\title{
COMPLEX CONVEXITY
}

\author{
BY \\ H. J. BREMERMANN(1)
}

1. Introduction. The first part of the present paper is a contribution to the theory of regions of holomorphy in $n$ complex dimensions. In particular one (characteristic) property of the regions of holomorphy is elaborated, namely, "pseudo-convexity." The methods developed here are adaptable to the study of holomorphic functionals in infinite dimensional Banach spaces (compare Bremermann [7]).

In the second part ( $\$ \$ 9 \mathrm{ff}$.$) we study the pseudo-convex regions and$ plurisubharmonic functions (introduced by K. Oka and P. Lelong, for definition see 2.6) for their own sake. We show that the notions "pseudo-convex" region and "plurisubharmonic function" are formal extensions of the notions "convex domain" and "convex function" from the $n$-dimesional vector space of $n$ real variables to the vector space $C^{n}$ of $n$ complex variables. While the convex functions, roughly speaking, are generated by linear functions, the plurisubharmonic functions are generated by analytic functions. Convex domains and pseudo-convex regions can be defined by means of convex functions and plurisubharmonic functions, respectively. Therefore the "complex convexity," by which notion we will denote pseudo-convex regions and plurisubharmonic functions jointly, is a more complicated property than the (real) convexity.

The basic theorem (5.1 and 5.4) of the first part is (simplified) the following: Let $H$ be a region of holomorphy. Let $S$ and $T$ be pointsets such that the closure of $S \cup T$ is in $H$ and such that every function which is holomorphic in $H$ takes its maximum modulus with respect to $\bar{S} \cup \bar{T}$ on $\bar{T}$. Let $\Delta_{H}(z)$ denote the distance of the point $z \in H$ from the boundary of $H$ measured in an arbitrary norm. Then the function $\Delta_{H}(z)$ takes its minimum with respect to $\bar{S} \cup \bar{T}$ already on $\bar{T}$. As consequences hereof we derive: A general "theorem of continuity," some results on the holomorph-convexity and the theorem: In a region of holomorphy $H$ the function $-\log \Delta_{H}(z)$ is for any norm a plurisubharmonic function in $H$. In regions of holomorphy (and only in regions of holomorphy) the functions $-\log \Delta_{H}(\mathrm{z})$ generate metric forms

$$
d s^{2}=\sum_{\mu, \gamma=1}^{n} \frac{\partial^{2}-\log \Delta_{H}(\zeta)}{\partial z_{\mu} \partial \bar{z}_{\nu}} d z_{\xi} d \bar{z}_{\nu}
$$

We compare these metrics with the metric generated by the (plurisubhar-

Presented to the Society, May 1, 1954; received by the editors September 26, 1955.

(1) Part of the work on this paper was supported by the Office of Naval Research under Contract N6ori-106, task order 5 at Stanford, California. 
monic) Bergman kernel function. Further we show: If for a region $D$ and a norm $N$ the function $-\log \Delta_{D}(z)$ is plurisubharmonic, then a certain "theorem of continuity" holds, and conversely, if the theorem of continuity holds, then any function $-\log \Delta_{D}(z)$, based on an arbitrary norm, is plurisubharmonic in $D$. A corollary hereof is: The property of the functions $-\log \Delta_{D}(z)$ to be plurisubharmonic for a region $D$ depends only upon the region but not upon the norm. The regions for which the functions $-\log \Delta_{D}(z)$ are plurisubharmonic we denote as "pseudo-convex regions." Thus every schlicht region of holomorphy is a pseudo-convex region (and the converse is also true according to a result of $\mathrm{K}$. Oka).

The pseudo-convex regions and plurisubharmonic functions are closely related to the convex domains and functions. The plurisubharmonic functions are formally defined by the same definition as the convex functions if one replaces " $R$ " by " $C^{n}$," "linear majorant" by "harmonic majorant," "straight line" by "analytic plane," etc. We demonstrate that many theorems hold for plurisubharmonic functions as well as for convex functions. The same is true for pseudo-convex regions and convex domains. A region $D \subset C^{n}$ is pseudoconvex if and only if the function $-\log \delta_{D}(z)$ where $\delta_{D}(z)$ is the euclidean distance function, is plurisubharmonic in $D$. We prove correspondingly: A domain $B \subset R^{n}$ is convex if and only if the function $-\log \delta_{B}(\mathfrak{x})$, where $\delta_{B}(\mathfrak{x})$ is the euclidean distance function of the domain $B$, is a convex function in $B$. We show that many corresponding theorems hold for pseudo-convex regions and convex domains and we derive a "restriction principle" which permits one to derive for a certain class of theorems on plurisubharmonic functions and pseudo-convex regions by restriction to tube domains the corresponding theorems for convex functions and domains.

The correspondence of theorems suggests that one interpret results of the theory of several complex variables in the convex case. We do this for Bergman's "extended class of functions" and obtain on the other hand from the interpretation a heuristic idea how to define a different "extended class" that serves the same purpose, namely to solve certain boundary value problems. We shall develop the details in a further paper.

Every domain $B C R^{n}$ possesses a convex envelope. The problem of defining the pseudo-convex envelope of regions $D \subset C^{n}$ leads one to consider nonschlicht regions. Plurisubharmonic functions can be defined on complex manifolds with no difficulty at all because the property of being plurisubharmonic is a local property and is not affected by holomorphic transformations. However, on complex manifolds there are no norms. Nevertheless one can define pseudo-convex regions in the following way: A region $D$ is pseudo-convex if and only if there exists a plurisubharmonic function that tends to infinity everywhere at the boundary of $D$. For schlicht regions this definition is equivalent to the one given before.

The methods developed here are suitable for infinite dimensional Banach 
spaces. There is one difficulty however, the infinite dimensional spaces are not locally compact. We shall show in a further paper that in spite of this difficulty several theorems of this paper can be extended (Bremermann [7]).

\section{Notation and auxiliary theorems.}

2.1. We consider the space $R^{n}$ of $n$ real variables $x_{1} \cdots x_{n}$ and the space $C^{n}$ of $n$ complex variables $z_{1} \cdots z_{n} ; z_{j}=x_{j}+i y_{j}$. We write for the real vector $\mathfrak{r}=\left(x_{1} \cdots x_{n}\right)$ and for the complex vector $z=\left(z_{1} \cdots z_{n}\right)$.

2.2. As topology we introduce the topology generated by the euclidean norm. We note that all topologies generated by arbitrary norms are equivalent. A region is an open set, a domain an open and connected set. Each subset of a region which is connected and cannot be enlarged without ceasing to be a subset of the region or ceasing to be connected, we call a component. Unless otherwise stated, we assume that our regions and domains are schlicht.

2.3. We write $\{z \mid P(z)\}$ for "the set of all $z \in C^{n}$ that satisfy the property $P(z)$." We use the symbol " $\wedge$ " for the logical conjunction.

2.4. Let $D$, be a sequence (or family) of regions such that $D_{\nu} \subset D$ and every point $z \in D$ belongs together with a fixed neighborhood to almost all $D_{\nu}$. Then we write " $\lim _{\nu \rightarrow \infty} D_{\nu}=D$."

2.5. A function $f(z)$ is holomorphic in a region $D$, if it is single-valued in $D$ and if for all $z \in D$ we have $\partial f(z) / \partial \bar{z}_{j}=0$ for $j=1, \cdots, n$. In other words, $f$ is holomorphic in each variable separately. We note that a consequence of this definition is that a holomorphic function is not determined by the values in one component. We are free to prescribe in the different disjoint components of a region "different" holomorphic functions and to consider them together as one holomorphic function in the region.

2.6. A function $V(\mathrm{z})$ is called "plurisubharmonic" $\left({ }^{2}\right)$ in a region $D$ if

(a) $-\infty \leqq V(z)<+\infty$ in $D$,

(b) $V(z)$ is upper semicontinuous in $D$,

(c) the Hermitian form

$$
\sum_{\mu, v=1}^{n} \frac{\partial^{2} V(z)}{\partial z_{\mu} \partial \bar{z}_{\nu}} d z_{\mu} d \bar{z}_{\nu}
$$

is positive semidefinite. (The differential form has to be taken in the sense of Schwartz (Schwartz [1]).) The condition (c) is equivalent to:

$\left(c^{\prime}\right)$ The function $V(z)$ is subharmonic in the intersection of every analytic

(2) This class of functions has been defined independently and at about the same time by Oka [1] and Lelong [1]. Also the "Hartogs"functions" of Bochner-Martin [1] are closely related to this class (compare Bremermann [5] and [6]). Oka called these functions "pseudoconvex functions." The author adopted in his paper Bremermann [1] the notation of Oka because he realized that the pseudo-convex domains correspond to the convex domains and the considered class of functions to the convex functions (which is discussed in detail in this paper). However, due to the work of Lelong, the denotation "plurisubharmonic functions" has become more widely known and accepted. 
plane $\left\{z \mid z=z^{(0)}+\lambda \mathfrak{a}\right\}$ with the region $D$ (a an arbitrary complex vector, $\lambda$ a complex parameter).

2.7. Let a function be holomorphic or plurisubharmonic in $D$ and upper semicontinuous in $\bar{D}$. Then it assumes its maximum on $\bar{D}-D$ unless it is a constant (Bremermann [1]).

2.8. A region $H$ is called a "region of holomorphy" if there exists a function holomorphic in $H$ and not holomorphic in a larger region (which could also be non-schlicht) (Behnke-Thullen [1]).

A region of holomorphy $H$ obviously has the property that it contains all points in which every function that is holomorphic in $H$ is still holomorphic.

2.9. TheOREM OF BeHNKe-Stein. If $\left\{H_{\nu}\right\}$ is a sequence of regions of holomorphy and $\lim _{\nu \rightarrow \infty} H_{\nu}=H$, then $H$ is a region of holomorphy (Behnke-Stein [1] and $O k a[1])$.

2.10. A region $D$ is called "holomurph-convex" if for every region $D_{0} \subset \subset D$ there exists a region $D^{*}, D_{0} \subset D^{*} \subset \subset D$, such that for every point $z^{(0)} \in D-\bar{D}^{*}$ there exists a function $f(z)$, holomorphic in $D$, such that

(Behnke-Thullen [1]).

$$
\max _{\mathfrak{z} \in D_{0}}|f(\mathfrak{z})|<\left|f\left(\mathfrak{z}^{(0)}\right)\right|
$$

2.11. Any schlicht region of holomorphy is holomorph-convex, and any holomorph-convex region is a region of holomorphy (Behnke-Thullen [1]).

2.12. A convex domain is a domain such that the straight line segment between any two of its points belongs to the domain.

A domain is convex if and only if through every boundary point passes a supporting straight line (plane, hyperplane) (Behnke-Stein [2]).

3. Properties of distance functions.

3.1. Let $N(z)$ be a norm in the complex vector space $C^{n}$ (for the real vector space $R^{n}$ the considerations are completely analogous). $N(z)$ is a norm if $N(z) \neq 0$ if $z \neq 0, N(0)=0, N\left(z^{(1)}+z^{(2)}\right) \leqq N\left(z^{(1)}\right)+N\left(z^{(2)}\right)$ and $N\left(\lambda_{z}\right)=|\lambda| N(z)$, $\lambda$ a complex scalar. In the following we shall write, if convenient, $\|z\|$ for $N(z)$.

The point set $\left\{z \mid\left\|z-z^{(0)}\right\|<r\right\}$ is the interior of the "sphere" of radius $r$ with center at $z^{(0)}$ with respect to the norm. Let $D$ be a region in the $C^{n}$, then we associate with every point $z \in D$ the value

$$
\Delta_{D}(z)={ }_{d f} \sup r \ni\left\{z^{\prime} \mid\left\|_{z}-z^{\prime}\right\|<r\right\} \subset D .
$$

That is the supremum of the radii of the spheres with center at $z$ that are contained in $D$, or in other words the distance of the point $z$ from the boundary of $D$ measured in the norm $N$. Thus $\Delta_{D}(z)$ is a real-valued function defined in $D$. We call $\Delta_{D}(z)$ the "distance function of $D$ based upon the norm $N$. "

3.2. In any region $D$ that is different from the $C^{n} \Delta_{D}(z)$ is continuous with respect to the topology generated by the norm. 
REMARK. As we have noted already the topology generated by an arbitrary norm is equivalent to the topology generated by the euclidean norm.

If $D$ is the whole $C^{n}$, then $\Delta_{D}(z) \equiv \infty$. If $D$ has at least one finite boundary point, then any point in $D$ has a finite distance from this point, and therefore $\Delta_{D}(z)$ is finite in $D$ in this case.

By definition we have: If $\left\|z-z^{(1)}\right\|<\Delta_{D}\left(z^{(1)}\right)$, then $z \in D$. If now $\left\|z^{(1)}-z^{(2)}\right\|$ $<\epsilon$, then because of the triangle inequality we have $\left\|z-z^{(1)}\right\|<\left\|z-z^{(2)}\right\|+\epsilon$. If therefore $\left\|_{z}-z^{(2)}\right\|<\Delta_{D}\left(z^{(1)}\right)-\epsilon$, then $z \in D$, thus by definition

$\Delta_{D}\left(z^{(2)}\right) \geqq \Delta_{D}\left(z^{(1)}\right)-\epsilon, \quad$ and for symmetry reasons $\Delta_{D}\left(z^{(1)}\right) \geqq \Delta_{D}\left(z^{(2)}\right)-\epsilon$.

Therefore we have

$$
\left|\Delta_{D}\left(z^{(1)}\right)-\Delta_{D}\left(z^{(2)}\right)\right|<\epsilon \text { if }\left\|z^{(1)}-z^{(2)}\right\|<\epsilon,
$$

hence $\Delta_{D}(z)$ is continuous in $D$ with respect to the norm.

3.3. For the intersection of regions we have, as we see from our definition,

$$
\Delta_{D_{1} \cap D_{2}}(\mathrm{z})=\min \left\{\Delta_{D_{1}}(\mathrm{z}), \Delta_{D_{2}}(\mathrm{z})\right\} \text {. }
$$

In general, if $D$ is the largest open set contained in the intersection of a family of regions $\left\{D_{\alpha}\right\}$, then $\Delta_{D}(z)$ is the lower envelope of the family of functions $\left\{\Delta_{D_{\alpha}}(z)\right\}$, that is

$$
\Delta_{D}(\mathfrak{z})=\inf _{\alpha}\left\{\Delta_{D_{\alpha}}(\mathfrak{z})\right\} .
$$

3.4. The distance function that belongs to the euclidean norm $\|z\|_{\text {e }}$ $=\left(\left|z_{1}\right|^{2}+\cdots+\left|z_{n}\right|^{2}\right)^{1 / 2}$ we denote by $\delta_{D}(z)$, and the distance function that belongs to the maximum norm $\left\|_{z}\right\|_{m}=\max _{j}\left\{\left|z_{j}\right|\right\}$ we denote by $\delta_{D}^{*}(z)$. (The function $\delta_{D}^{*}(z)$ was introduced by Cartan-Thulleh [1] and termed "Randdistanz".) We note that a distance function is invariant with respect to the same transformations of a domain $D$ with respect to which the norm is invariant. Thus $\delta_{D}(z)$ is invariant with respect to euclidean transformations and $\delta_{D}^{*}(z)$ is invariant with respect to the group of permutations of the coordinate planes and euclidean transformations within the planes.

3.5. Let $\mathfrak{a}$ be a complex vector of norm one, $\|\mathfrak{a}\|=1$, and $\lambda$ a complex scalar. Then we define

$$
\Delta_{\mathfrak{a}, D}(\mathfrak{z})={ }_{d f} \sup r \ni\left\{z^{\prime}\left|z^{\prime}=\mathfrak{z}+\lambda \mathfrak{a} \wedge\right| \lambda \mid<r\right\} \subset D,
$$

in other words $\Delta_{a, D}(z)$ is the radius (measured in the norm $N$ ) of the largest disk with center $z$ on the analytic plane $\left\{z^{\prime} \mid z^{\prime}=z+\lambda \mathfrak{a}\right\}$ that is contained in $D$.

When $a$ varies through all directions, then the union of all circles $\left\{z^{\prime} \mid z^{\prime}\right.$ $=z+\lambda a \wedge|\lambda|<r\}$ is obviously equal to the sphere $\left\{z^{\prime} \mid\left\|z-z^{\prime}\right\|<r\right\}$. Therefore we have

$$
\Delta_{D}(z)=\inf _{a}\left\{\Delta_{a, D}(z)\right\}
$$


4. The maximum principle.

4.1. We say that the "maximum principle" holds for the point sets $S$ and $T$ with respect to the class of (real-valued) functions $\mathcal{L}$, if for any function $V(z) \in \mathcal{L}$ we have the equality

$$
\sup _{z \in T} V(z)=\sup _{z \in S \cup T} V(z) .
$$

Analogously we say "the minimum principle" holds for $S, T$, and $\mathcal{L}$.

4.2. Let $F$ be a $2 k$-dimensional analytic surface $(1 \leqq k \leqq n)$ with a parameter representation and only ordinary points. That is, for every point $z^{(0)} \in F$ there exists a vector function $h_{8}{ }^{(0)}\left(\lambda_{1}, \cdots, \lambda_{k}\right)$ such that $z^{(0)}=h_{8}{ }^{(0)}\left(\lambda_{1}^{(0)}, \cdots\right.$, $\left.\lambda_{k}^{(0)}\right)$ and that $h_{z}^{(0)}\left(\lambda_{1}, \cdots, \lambda_{k}\right)$ is holomorphic in a neighborhood of $\lambda_{1}^{(0)}, \cdots, \lambda_{k}^{(0)}$ and $z=h_{b}{ }^{(0)}\left(\lambda_{1}, \cdots, \lambda_{k}\right)$ represents $F$ in a neighborhood of $z^{(0)}$, and the functional determinant is of rank $k$.

If an analytic surface $F$ is contained in a domain $D$ and $f(z)$ is a function, holomorphic in $D$, then $f\left(h_{3}^{(0)}\left(\lambda_{1}, \cdots, \lambda_{k}\right)\right)$ is for every $z^{(0)}$ a holomorphic function of $\lambda_{1}, \cdots, \lambda_{k}$ in a neighborhood of $\lambda_{1}^{(0)}, \ldots, \lambda_{k}^{(0)}$. We can say " $f(z)$ is holomorphic on $F$." However, in order to consider functions "holomorphic on $F$ " it is not necessary that $f(z)$ is defined in a full $2 n$-dimensional neighborhood of $F$. We call $f(z)$ "holomorphic on $F^{\text {" if }} f(z)$ is defined and singlevalued on $F$ and if $f\left(h_{8}{ }^{(0)}\left(\lambda_{1}, \cdots, \lambda_{k}\right)\right)$ is holomorphic for every $z^{(0)} \in F$ in a neighborhood of $\lambda_{1}^{(0)}, \cdots, \lambda_{\mathbf{k}}^{(0)}$.

4.3. Let $S$ be a domain on an analytic surface $F$ and $T$ its boundary and $S \cup T \subset F$. Then the maximum principle holds for the class of moduli of functions that are holomorphic on $S$ and continuous on $S \cup T$.

Proof. According to our Definition 4.1 the maximum principle holds if $f(z)$ is a constant. Suppose there would exist a function $f(z)$ holomorphic on $S$ and continuous on $S \cup T$ for which the maximum principle is violated. Let $m$ be the maximum of $|f(z)|$ in $S \cup T$ and $M$ the point set in $S \cup T$ for which $|f(z)|=m$. Obviously $M \subset C S$. Let $z^{(0)}$ be a boundary point of $M$. Then $f\left(h_{0}{ }^{(0)}\left(\lambda_{1}, \cdots, \lambda_{k}\right)\right)$ would take its maximum at an interior point of a neighborhood of $\left(\lambda_{i}^{(0)}, \cdots, \lambda_{k}^{(0)}\right)$, and would not be a constant. That is a contradiction.

4.4. If an analytic surface $F$ is contained in a domain $D$ and $V(z)$ is plsh. in $D$, then $V\left(h_{\jmath^{(0)}}\left(\lambda_{1}, \cdots, \lambda_{k}\right)\right)$ is for every $z^{(0)} \in F$ a plsh. function in a neighborhood of $\lambda_{1}^{(0)}, \cdots, \lambda_{k}^{(0)}$. We say " $V(\mathfrak{z})$ is plsh. on $F$." Analogously as in 4.3 we define "a function $V(z)$ is plsh. on $F$ " if $V(z)$ is defined on $F$ and if $V\left(h_{b^{(0)}}\left(\lambda_{1}, \cdots, \lambda_{k}\right)\right)$ is plurisubharmonic for every $z^{(0)} \in F$ in a neighborhood of $\lambda_{1}^{(0)}, \cdots, \lambda_{k}^{(0)}$.

4.5. Let $S$ be a domain on an analytic surface $F$ and $T$ its boundary and $S \cup T \subset F$. Then the maximum principle holds for the class of functions that are plsh. on $S$ and upper semi-continuous on $S \cup T$.

The proof goes exactly as in 4.3 because a plurisubharmonic function, 
too, takes its maximum at an interior point of a domain where it is plsh. if and only if it is a constant (see 2.7).

4.6. As the moduli of holomorphic functions are special plurisubharmonic functions, the maximum principle holds for $S, T$ and the class of functions that are holomorphic in a neighborhood of $\bar{S} \cup \bar{T}$ whenever it holds for $S, T$ and the class of functions that are plurisubharmonic in a neighborhood of $\bar{S} \cup \bar{T}$.

The converse is true in special cases (as in 4.3). Also it has been conjectured by Bochner and Martin that every plurisubharmonic function is a Hartogs function from which the converse would have followed. However it has been shown in Bremermann [5] and [6] that the conjecture is not true in general and the counterexample constructed in [5] is also an example of a pair of sets for which the maximum principle holds with respect to the moduli of holomorphic functions but not with respect to plurisubharmonic functions.

The converse is true for the classes of functions that are holomorphic, plurisubharmonic respectively, in a fixed domain $D$ such that $S \cup T \subset \subset D$, where $D$ is a domain of holomorphy (Bremermann [5]). It is an open problem to decide whether the converse is true in this case only when $D$ is a domain of holomorphy. The general question of the characterisation of the sets for which the maximum principle holds for holomorphic functions seems to be a deep one. (Compare also Behnke-Sommer [1].)

5. Distance functions in regions of holomorphy.

5.1. Theorem. (Compare Bremermann [4, §4], and Cartan-Thullen [1].) Let $H$ be a region of holomorphy. Let the maximum principle be valid for the point sets $S, T$ with respect to the class of the moduli of the functions that are holomorphic in a region $D$ such that $\bar{S} \cup \bar{T} \subset D \subset H$. Let $\chi(z)$ be an arbitrary function holomorphic and different from zero in $D$. Let $S$ be an open set. Then we have

(1) $\inf _{\mathfrak{z} \in T}|\chi(z)| \Delta_{a, H}(z)=\inf _{\mathfrak{z} \in S \cup T}|\chi(z)| \Delta_{a, H}(z)$ and

(2) $\inf _{\mathrm{z} \in T}|\chi(z)| \Delta_{H}(z)=\inf _{\mathrm{z} \in S \cup T}|\chi(z)| \Delta_{H}(z)$,

where $\Delta_{a, H}(\xi)$ and $\Delta_{H}(\xi)$ are distance functions as defined in $\S 3$ based upon an arbitrary norm.

REMark. The methods developed here are adaptable to the case of infinite-dimensional Banach and Hilbert spaces. We shall, however, treat the infinite-dimensional case in a further paper (Bremermann [7]).

We shall prove the theorem under the assumption that $S$ is an open set. Later, in 5.4 we shall free ourselves from this assumption in the case of the second equality.

Proof. The second equation follows from the first: It is

$$
\inf _{\|a\|=1} \inf _{\mathfrak{z} \in T}|\chi(z)| \Delta_{\mathfrak{a}, H}(z)=\inf _{\mathfrak{z} \in T} \inf _{\|a\|=1}|\chi(z)| \Delta_{\mathfrak{a}, H}(z)=\inf _{\mathfrak{z} \in T}|\chi(z) .| \Delta_{H}(z)
$$

where inf $\|a\|_{-1}$ means the infimum taken with respect to all vectors $\mathfrak{a}$ with 
norm one. And just the same we have

$$
\begin{aligned}
\inf _{\|a\|=1} \inf _{z \in S \cup T}|\chi(z)| \Delta_{a, H}(z) & =\inf _{\mathfrak{z} \in S \cup T} \inf _{\|a\|=1}|\chi(z)| \Delta_{a, H}(z) \\
& =\inf _{\mathfrak{z} \in S \cup T}|\chi(z)| \Delta_{H}(z) .
\end{aligned}
$$

Therefore the second equality is proved when the first equality is proved.

Proof of the first equality of 5.1: We choose a region $H^{*}$ such that $\bar{S} \cup \bar{T} \subset H^{*} \subset \subset H$. Such an $H^{*}$ exists because we have by assumption $\bar{S} \cup \bar{T}$ $\subset H$. Because $\bar{T} \subset H^{*}, \inf _{z \in T} \Delta_{a, H^{*}(z)}|\chi(z)|=m$ exists and is larger than zero. Let $\mathfrak{a}$ be a complex vector with $\|\mathfrak{a}\|=1$ and $\lambda$ a complex scalar. Then the set

$$
T^{*}={ }_{d f}\left\{\left.z\left|z^{\prime} \in T \wedge z=z^{\prime}+\lambda \mathfrak{a} \wedge\right| \lambda|<m| \chi\left(z^{\prime}\right)\right|^{-1}\right\}
$$

is a subset of $H^{*}$.

Let $f(z)$ be a function holomorphic in $H$ that cannot be continued into a domain larger than $H$. Such a function exists because $H$ is by assumption a region of holomorphy. Because $H^{*} \subset \subset \mathrm{C}$ there exists an $M$ such that $|f(z)|<M$ in $H^{*} . f\left(z^{\prime}+\lambda a\right)$ is for fixed $z^{\prime}$ a holomorphic function of $\lambda$ in the intersection of $H$ with the analytic plane $\left\{z \mid z=z^{\prime}+\lambda \mathfrak{a}\right\}$. We develop $f\left(z^{\prime}+\lambda \mathfrak{a}\right)$ in a Taylor series:

$$
f\left(z^{\prime}+\lambda \mathfrak{a}\right)=\left.\sum_{\nu=0}^{\infty} \frac{1}{\nu !} \frac{\partial^{\nu} f\left(z^{\prime}+\lambda \mathfrak{a}\right)}{\partial \lambda^{\nu}}\right|_{\lambda=0} \lambda^{\nu} .
$$

If $z^{\prime} \in T$ and $|\lambda|<m\left|\chi\left(z^{\prime}\right)\right|^{-1}$, then $z^{\prime}+\lambda \mathfrak{a} \in T^{*} \subset H^{*}$, therefore $\left|f\left(z^{\prime}+\lambda \mathfrak{a}\right)\right|$ $<M$. Therefore, applying Cauchy's formula we obtain

$$
\left|\frac{1}{\nu !} \frac{\partial^{\nu} f\left(z^{\prime}+\lambda \mathfrak{a}\right)}{\partial \lambda^{\nu}}\right|_{\lambda=0} \mid \leqq \frac{M}{m^{\nu}\left|\chi\left(z^{\prime}\right)\right|^{-\nu}}, \quad \text { for } z^{\prime} \in T,
$$

hence

$$
\left.\left|\frac{1}{\nu !} \frac{\partial^{\nu} f\left(\mathfrak{z}^{\prime}+\lambda \mathfrak{a}\right)}{\partial \lambda^{\nu}}\right|_{\lambda=0}|| \chi\left(z^{\prime}\right)\right|^{-\nu} \leqq \frac{M}{m^{\nu}} \quad \text { for } z^{\prime} \in T .
$$

The left-hand side is a holomorphic function in $D$. Therefore, applying that for $S, T$ and the functions that are holomorphic in $D$ the maximum principle holds, we conclude that the latter inequality holds not only for $z^{\prime} \in T$ but for $z^{\prime} \in S$.

Therefore the series

$$
\left.\sum_{\nu=0}^{\infty} \frac{1}{\nu !} \frac{\partial^{\nu} f\left(z^{\prime}+\lambda \mathfrak{a}\right)}{\partial \lambda^{\nu}}\right|_{\lambda=0} \lambda^{\nu}
$$

converges uniformly in any point set

$$
\left\{\left.z\left|z^{\prime} \in S \wedge z=z^{\prime}+\lambda \mathfrak{a} \wedge\right| \lambda|\leqq \vartheta m| \chi\left(z^{\prime}\right)\right|^{-1}\right\}
$$


where $0<\vartheta<1$. The series is a generalized Hartog series (compare Bremermann [1]). It represents our function $f(z)$ if the set (3) is an open set. This is ensured if $S$ is an open set.

Letting $\vartheta \rightarrow 1$ we conclude that $f(z)$ is holomorphic in the set

$$
\left\{\left.z\left|z^{\prime} \in S \wedge z=z^{\prime}+\lambda \mathfrak{a} \wedge\right| \lambda|<m| \chi\left(z^{\prime}\right)\right|^{-1}\right\} .
$$

$f(z)$ is by assumption not holomorphic in any domain larger than $H$. Therefore this pointset belongs to $H$. Hence

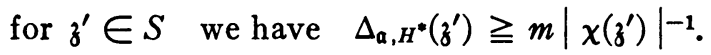

This is true for every $H^{*}$. Letting $H^{*} \rightarrow H$, then

$$
m=\inf _{\mathfrak{z} \in T} \Delta_{\mathfrak{a}, H^{*}}(\mathfrak{z})|\chi(\mathfrak{z})|^{-1} \rightarrow \inf _{\mathfrak{z} \in T} \Delta_{\mathfrak{a}, H}(\mathfrak{z})|\chi(z)|^{-1} .
$$

Therefore

$$
\inf _{\mathfrak{s} \in S} \Delta_{a, H}(z)|\chi(z)|^{-1} \geqq \inf _{\mathfrak{z} \in T} \Delta_{a, H}(z)|\chi(z)|^{-1},
$$

from where the first equality of the theorem follows immediately. q.e.d.

5.2. REMARK. The proof of Theorem 5.1 works out just the same if instead of the "strong maximum principle": If $V(z)<M$ on $T$, then $V(z)<M$ on $S$ we have only the following "weak maximum principle." If $V(z)<M$ on $T$, then $V(z)<c M$, where $c$ is a constant independent of the particular function $V(z)$. Indeed, from inequality (1) in the proof of 5.1 we get

$$
\left.\left|\frac{1}{\nu !} \frac{\partial^{\nu} f\left(z^{\prime}+\lambda \mathfrak{a}\right)}{\partial \lambda^{\nu}}\right|_{\lambda=0}|| \chi\left(z^{\prime}\right)\right|^{-1} \leqq \frac{c \cdot M}{m^{\nu}} \quad \text { for } z^{\prime} \in S
$$

(instead of $\leqq M / m^{\nu}$ ). But this inequality is sufficient to conclude that the series (2) converges uniformly in any point set (3). Hence Theorem 5.1 is true also if the strong maximum principle is replaced by the weak maximum principle.

5.3. Lemma. Let the maximum principle be valid for the point sets $S, T$ and the class $\mathcal{L}$ of moduli of functions that are holomorphic in a fixed open set $D$ containing $\bar{S} \cup \bar{T}$. Let

$$
T(\rho)={ }_{d f}\left\{z \mid z^{\prime} \in T \wedge\left\|z^{\prime}-z\right\| \leqq \rho\right\}
$$

and

$$
S(\rho)={ }_{d f}\left\{z \mid z^{\prime}=S \wedge\left\|z^{\prime}-z\right\| \leqq \rho\right\} .
$$

Let $\rho_{0}$ be such that $T\left(\rho_{0}\right) \cup S\left(\rho_{0}\right) \subset D$ for $\rho \leqq \rho_{0}$. If $f(z) \in \mathcal{L}$ and $|f(z)| \leqq M$ in $T\left(\rho_{0}\right)$, then $|f(z)| \leqq 2 M$ in $S\left(\rho_{0} / 2\right)$.

Proof. Let $z=z^{\prime}+\lambda \mathfrak{a}, \lambda$ a complex scalar and $\|\mathfrak{a}\|=1$. Let $f \in \mathcal{L}$. If then $z^{\prime} \in S \cup T$ and $|\lambda|<\rho_{0}$, then $z \in T\left(\rho_{0}\right) \cup S\left(\rho_{0}\right) \subset D$ and we have for $f\left(z^{\prime}+\lambda a\right)$ the development 


$$
f\left(\mathfrak{z}^{\prime}+\lambda \mathfrak{a}\right)=\left.\sum_{\nu=0}^{\infty} \frac{1}{\nu !} \frac{\partial^{\nu} f\left(\mathfrak{z}^{\prime}+\lambda \mathfrak{a}\right)}{\partial \lambda^{\nu}}\right|_{\lambda=0} \lambda^{\nu}
$$

If now $|f(z)|<M$ in $T\left(\rho_{0}\right)$, then we have by Cauchy's formula

$$
\text { for } z^{\prime} \in T: \quad\left|\frac{1}{\nu !} \frac{\partial^{\nu} f\left(z^{\prime}+\lambda a\right)}{\partial \lambda^{\nu}}\right|_{\lambda=0} \mid \leqq \frac{M}{\rho_{0}{ }^{\nu}} .
$$

Because the maximum principle holds for $S$ and $T$, the last inequality holds also for $z^{\prime} \in S$. Therefore

$$
f\left(\mathfrak{z}^{\prime}+\lambda \mathfrak{a}\right) \leqq M \sum_{\nu=0}^{\infty}\left(\frac{|\lambda|}{\rho_{0}}\right)^{\nu} .
$$

For $|\lambda| \leqq \rho_{0} / 2$ the value of the series is smaller than or equal to 2 . Letting the vector $a$ vary through all directions we obtain

$$
f(z) \mid \leqq 2 M \text { for } z \in S\left(\rho_{0} / 2\right) .
$$

q.e.d.

5.4. Theorem 5.1.2 is valid without the additional assumption that $S$ is an open set.

Proof. Let the maximum principle be valid for $S, T$, but let $S$ be arbitrary otherwise. Then we apply Theorem 5.1 to $S(\rho / 2)$ and $T(\rho)$. For $S(\rho / 2)$ and $T(\rho)$ the weak maximum principle holds and $S(\rho / 2)$ is an open set. Therefore by 5.2 we obtain

$$
\inf _{z \in T(\rho)} \Delta_{H}(z)|\chi(z)|=\inf _{z \in S(\rho / 2) \cup T(\rho)} \Delta_{H}(z)|\chi(z)| \quad \text { for } \rho \leqq \rho_{0} .
$$

Letting $\rho \rightarrow 0$, then $S(\rho / 2)$ tends to $S$ and $T(\rho)$ to $T$, and the infimum with respect to $T(\rho) \cup S(\rho / 2)$ tends to the infimum with respect to $T \cup S$, because $\Delta_{H}(z)$ is continuous; and $\inf _{z \in T(\rho)}$ tends to $\inf _{z \in T}$. Therefore we obtain

$$
\inf _{z \in T} \Delta_{H}(z)|\chi(z)|=\inf _{z \in S \cup T} \Delta_{H}(z)|\chi(z)| . \quad \text { q.e.d. }
$$

6. The theorem of continuity and the holomorph-convexity as consequences of the "Fundamental theorem."

6.1. Theorem of Continuity ("Kontinuitätssatz"). (Compare BehnkeSommer [1] and Behnke-Stein [3].) Let $H$ be a region of holomorphy. Let $\left\{S_{\nu}\right\}$, $\left\{T_{\nu}\right\}$ be sequences of sets, such that $S_{\nu} \cup T_{\nu} \subset \subset C_{1}$ and such that the maximum principle is valid for every $\nu$ for $S_{\nu}, T_{\nu}$ and the class of moduli of functions that are holomorphic in H. Let $\lim _{\nu \rightarrow \infty} S_{\nu}=S_{0}$ and $\lim _{\nu \rightarrow \infty} T_{\nu}=T_{0}$ and let $S_{0} \cup T_{0}$ be bounded.

Then $T_{0} \subset \subset H$ implies $S_{0} \cup T_{0} \subset \subset H$.

Proof. Applying Theorem 5.1.2 for the euclidean distance function and $\chi(z) \equiv 1$ we obtain 


$$
\inf _{\mathfrak{z} \in T_{\nu}} \delta_{H}(z)=\inf _{\mathfrak{z} \in T_{\nu} \cup S_{\nu}} \delta_{H}(z) \text {. }
$$

As we have proved in 3.2 the distance function $\delta_{H}(z)$ is continuous in $H$. Also $S_{0} \cup T_{0}$ is bounded. Therefore this relation holds also in the limit, that means

$$
\inf _{\jmath \in T_{0}} \delta_{H}(z)=\inf _{\jmath \in T_{0} \cup S_{0}} \delta_{H}(z) \text {. }
$$

Because $T_{0} \subset \subset H$ we have $\inf _{z \in T_{0}} \delta_{H}(z)>0$, and therefore $\inf _{\mathrm{z} \in T_{0} \cup s_{0}} \delta_{H}(z)$ $>0$, and therefore

$$
S_{0} \cup T_{0} \subset \subset H .
$$

q.e.d.

6.2. Remark. A basic difference between one and several complex variables is the following: In one variable the sets $S, T$ for which the maximum principle holds for the moduli of holomorphic functions are essentially subdomains of $H$ and their boundaries. The condition that the interior points of a subdomain of a domain of holomorphy $H$ have a distance from the boundary of $H$ greater than or equal to the distance of its boundary is a trivial one and does not impose any restrictions on $H$. Any domain is a domain of holomorphy. In several complex variables, however, there are the 2-dimensional analytic surfaces for which the maximum principle holds (see 4.4). It is not trivial that the interior points of an analytic surface in a domain $H$ have a larger distance from the boundary of $H$ than the points of the boundary (of the analytic surface). Therefore for $n>1$ the validity of our fundamental theorem and the theorem of continuity is a condition on $H$ which is satisfied only by a certain class of domains.

6.3. HOLOMORPH CONVEXITy. The holomorph convexity of regions of holomorphy is well known (see 2.10 and 2.11). However, we should like to show that it can be easily derived from our fundamental Theorem 5.1 (5.4). We formulate the following theorems of this section for the euclidean distance function, but they are valid for an arbitrary distance function $\Delta_{H}(\mathrm{z})$ just as well.

Let $H$ be a bounded region of holomorphy. Let $H_{0}$ be a subregion, $H_{0} \subset \subset H$. Let $\inf _{\mathfrak{z} \in H_{0}} \delta_{H}(\mathfrak{z})=m$. Let

$$
H^{*}=\left\{z \mid z \in H \wedge \delta_{H}(z) \geqq m\right\} .
$$

Then there exists to every point $z^{(0)} \in H-H^{*}$ a function $f(z)$, holomorphic in $H$, such that

$$
\left|f\left(z^{(0)}\right)\right|>\sup _{z \in H^{*}}|f(z)| \geqq \sup _{\mathfrak{z} \in H_{0}}|f(z)| .
$$

Proof. Suppose this would not be true. Then for any function $f(z)$ holomorphic in $H$ we would have 


$$
\left|f\left(z^{(0)}\right)\right| \leqq \sup _{z \in H^{*}}|f(z)| \text {. }
$$

Therefore, if we put $S=\left\{z^{(0)}\right\}$ and $T=H^{*}$, the maximum principle holds for $S, T$, and the moduli of the functions holomorphic in $H$. That $S \subset \subset H$ is trivial and $T \subset \subset H$ because $H$ is bounded. Therefore we have according to our Theorems 5.1 and 5.4.

$$
\delta\left(z^{(0)}\right) \geqq \inf _{z \in T} \delta(z)=m .
$$

Therefore $z^{(0)} \in H^{*}$, but that is a contradiction to the fact that we have chosen $z^{(0)} \in H-H^{*}$.

6.4. Let $H$ be a bounded region of holomorphy, let $\chi(\xi)$ be a function holomorphic, bounded, and different from zero in $H$. Let

$$
H(\chi(z), \rho)=\left\{z\left|z \in H \wedge \delta_{H}(z)\right| \chi(z) \mid>\rho\right\} .
$$

Then for every point $z^{(0)} \in H-\bar{H}(\chi(z), \rho)$ there exists a function, holomorphic in $H$ such that

$$
\left|f\left(z^{(0)}\right)\right|>\sup _{z \in H(x(z), p)}|f(z)| .
$$

The region $H(\chi(z), \rho)$ is compact in $H$ because $H$ and $\chi(z)$ are bounded. Using Theorem 5.1 we conclude exactly as in 6.3.

6.5. Under the assumptions of the preceding Theorem 6.4 any region $H(\chi(z), \rho)$ is "convex with respect to functions that are holomorphic in $H$. " That means: For any compact subregion $H_{0}$ of $H(\chi(z), \rho)$ there exists a region $H^{*}$ such that

$$
H_{0} \subset H^{*} \subset \subset H(\chi(z), \rho)
$$

and to every point $z^{(0)}$ in $H(\chi(z), \rho)-\bar{H}^{*}$ there exists a function $f(z)$ holomorphic not only in $H(\chi(\xi), \rho)$ but in $H$ such that

$$
\left|f\left(z^{(0)}\right)\right|>\sup _{z \in H^{*}}|f(z)| .
$$

Indeed, any region $H\left(\chi(z), \rho^{\prime}\right)$ where $\rho^{\prime}$ is such that $H_{0} \subset H\left(\chi(z), \rho^{\prime}\right)$ $\subset \subset H(\chi(z), \rho)$ will satisfy this condition according to 6.4 , and there always exist $\rho^{\prime}$.

6.6. Under the assumptions of 6.4 any region $H(\chi(\xi), \rho)$ is a region of holomorphy. This follows from 6.5 and a theorem by Cartan-Thullen: Any region that is holomorph-convex is a region of holomorphy, (2.11).

7. $-\log \Delta_{H}(z)$, plurisubharmonic functions, metric forms, and kernel functions in regions of holomorphy.

7.1. In a region of holomorphy any function $-\log \Delta_{H}(z)$ is a plurisubharmonic function. (For the definition of the plsh. functions, see 2.6.) 
This result has first been proved for $-\log \delta_{H}(z)$ by the author in the following way (Bremermann [1, p. 54], compare also Lelong [3]): We show that $-\log \delta_{H}(z)$ is plsh. in regions that satisfy a certain theorem of continuity (compare also 8.4). As any region of holomorphy satisfies this theorem of continuity, $-\log \delta_{H}(z)$ is plsh. in regions of holomorphy. 5.4).

We shall give here a simpler proof based directly on Theorem 5.1 (and

Suppose it would not be true. Then, as $-\log \Delta_{H}(z)$ is continuous, there would exist an analytic plane $\left\{z \mid z=z^{(0)}+\lambda a\right\}$ such that $-\log \Delta_{H}(z)$ is not subharmonic in $\left\{z \mid z=z^{(0)}+\lambda a\right\} \cap H$ (compare Definition 2.6). That means there exists a point $z^{(1)}$ on the plane and a (small) circle around $z^{(1)}$ such that $-\log \Delta_{H}\left(\mathfrak{z}^{(0)}+\lambda \mathfrak{a}\right)$ is not subharmonic in the circle. For simplicity's sake we change the representation of the analytic plane to $\left\{z \mid z^{(1)}+\lambda \mathfrak{a}\right\}$. Let the radius of the circle be $r$ and let $V(\lambda)=-\log \Delta_{H}\left(\mathfrak{z}^{(1)}+\lambda \mathfrak{a}\right)$.

Then there exists a function $h(\lambda)$, harmonic for $|\lambda|<r$, and continuous for $|\lambda| \leqq r$, such that

$$
V(\lambda) \leqq h(\lambda) \text { for }|\lambda|=r
$$

and there exists a $\lambda^{(0)}$ such that

$$
V\left(\lambda^{(0)}\right)>h\left(\lambda^{(0)}\right) .
$$

As the circle $|\lambda|<r$ is simply connected we find a conjugate function $h^{*}(\lambda)$ such that $h(\lambda)+i h^{*}(\lambda)$ is holomorphic for $|\lambda|<r$. We approximate the function $h(\lambda)+i h^{*}(\lambda)$ by a function $g(\lambda)$ that is holomorphic in the whole $\lambda$-plane such that the inequalities (1) and (2) are still valid for the real part of $g(\lambda)$. Then we continue $g(\lambda)$ holomorphically into the whole space. This can easily be done by prescribing $g(z)=g(\lambda)$ in all points $z=z^{(1)}+\lambda a$ $+\rho_{2} \mathfrak{b}^{(2)}+\cdots+\rho_{n} \mathfrak{b}^{(n)},\left|\rho_{2}\right|<\infty, \cdots,\left|\rho_{n}\right|<\infty$, where the $\mathfrak{b}^{(j)}$ are such that $\left\{\mathfrak{a}, \mathfrak{b}^{(2)} \ldots \mathfrak{b}^{(n)}\right\}$ is an orthonormal base of the space $C^{n}$. Then $g(z)$ is holomorphic in the $C^{n}$, and therefore in particular holomorphic in $H$.

Let

$$
S=\left\{z\left|z=z^{(1)}+\lambda \mathfrak{a} \wedge\right| \lambda \mid<r\right\}
$$

and

$$
T=\left\{z\left|z=z^{(1)}+\lambda \mathfrak{a} \wedge\right| \lambda \mid=r\right\} .
$$

Then the maximum principle holds for $S$ and $T$. Therefore we should have according to Theorem 5.1 and Remark 5.4:

$$
\Delta_{H}(z)\left|e^{o(z)}\right| \geqq \inf _{z \in T} \Delta_{H}(z)\left|e^{o(z)}\right| \quad \text { for } z \in S .
$$

We have however

$$
V(\lambda) \leqq \operatorname{Re} g(z)
$$

on $T$ 
which is equivalent to

$$
0 \leqq \log \left(\Delta_{H}(z)\left|e^{o(z)}\right|\right) \quad \text { on } T
$$

which is equivalent to

$$
1 \leqq \Delta_{H}(z)\left|e^{g(z)}\right|
$$

on $T$.

On the other hand we have at the point $z^{(2)}=z^{(1)}+\lambda^{(0)} \mathfrak{a}$,

$$
1>\Delta_{H}\left(z^{(2)}\right)\left|e^{g(z(z))}\right| \text {. }
$$

$z^{(2)}$ is a point in $S$. That is a contradiction. Therefore $-\log \Delta_{H}(z)$ is plurisubharmonic in $H$.

7.2. CoROLlaRy. If $H$ is a region of holomorphy, then there exists a function $V(z)$ that is plurisubharmonic in $H$ and that tends to infinity everywhere at the boundary of $H$. We say " $V(z)$ tends to infinity everywhere at the boundary of $H$ " if for arbitrary large $M$ we have $\{z \mid z \in H \wedge V(z)<M\} \subset \subset H$.

The function $-\log \Delta_{H}(z)$ is plurisubharmonic in $H$ as we have just proved and it tends to infinity at every finite boundary point. Therefore if $H$ is bounded, then $-\log \Delta_{H}(\xi)$ has the required properties. If $H$ is not bounded, the function $-\log \Delta_{H}(z)$ may remain finite when approaching an infinite boundary point of $H$ (examples can easily be constructed). In this case we take $-\log \Delta_{H}^{*}(z)=_{d f} \sup \left\{-\log \Delta_{H}(z), \log \left|z_{1}\right|, \cdots, \log \left|z_{n}\right|\right\}$. The functions $\log \left|z_{1}\right| \cdots \log \left|z_{n}\right|$ are plurisubharmonic and the upper envelope of a finite set of plurisubharmonic functions is plurisubharmonic. $-\log \Delta_{H}^{*}(z)$ tends to infinity everywhere at the boundary, even if $H$ is unbounded, therefore it has the required properties and our corollary is proved.

7.3. The Corollary 7.2 is not really a weaker statement than 7.1 because we shall show later (in 9.25): If $D$ is a region such that there exists a function $V(z)$ that is plurisubharmonic in $D$ and becomes infinite everywhere at the boundary, then for any norm $-\log \Delta_{D}(z)$ is plurisubharmonic in the region.

7.4. A function $V(z)$ is plurisubharmonic if and only if the Hermitian form

$$
\sum_{\mu, v=1}^{n} \frac{\partial^{2} V}{\partial z_{\mu} \partial \bar{z}_{\nu}} d z_{\mu} d \bar{z}_{\nu}
$$

is positive semidefinite. (If $V(z)$ is not differentiable in the classical sense we have to take the form in the sense of Schwartz's distribution.) (See 2.6.) However, any plurisubharmonic function can be approximated uniformly in any compact subregion by plurisubharmonic functions that are arbitrarily differentiable in the classical sense such that the form is positive definite (Bremermann [1]).

Thus in regions of holomorphy (and only in regions of holomorphy, compare 8.7) the functions $-\log \Delta_{H}(z)$ generate metric forms 


$$
d s^{2}=\sum_{\mu, \nu=1}^{n} \frac{\partial^{2}-\log \Delta_{H}\left(z^{2}\right)}{\partial z_{\mu} \partial \bar{z}_{\nu}} d z_{\mu} d \bar{z}_{\nu}
$$

( $d s^{2}$ is only positive semidefinite, however).

The metric form generated by $-\log \delta_{H}(z)$ is invariant with respect to euclidean transformations of $H$, because $\delta_{H}(z)$ is euclidean invariant. (Compare 3.4.) The metric form generated by $-\log \delta_{H}^{*}(z)$ is invariant with respect to the permutations of the coordinate planes and euclidean transformations within the planes (compare 3.4).

7.5. In connection with the preceding considerations we remember that the metric form, generated by the Bergman kernel function,

$$
d s^{2}=\sum_{\mu, \nu=1}^{n} \frac{\log K_{D}\left(z_{,}, \bar{z}\right)}{\partial z_{\mu} \partial \bar{z}_{\nu}} d z_{\mu} d \bar{z}_{\nu},
$$

is holomorphically invariant, and $\log K_{D}(\bar{z}, \bar{z})$ is plsh. in $D$ (Bergman [2; $3 ; 5]$, Bremermann [2]).

However, $\log K_{D}(z, \bar{z})$ is not itself invariant with respect to holomorphic transformations, the transformation law is

$$
K_{D^{*}}\left(z^{*}, \bar{z}^{*}\right)=K_{D}(\bar{z}, \bar{z})|\operatorname{Det}| \frac{\partial z}{\partial z^{*}}||^{2} \text {. }
$$

The metric form has the special feature that it is not only semidefinite but definite and that its coefficients are real analytic.

The question arises: Does $\log K_{D}(z, \bar{z})$ become infinite everywhere at the boundary of $D$ ?

We have studied this question in Bremermann [2]. We obtained: $A$ necessary condition that $\log K_{D}(z, \bar{z})$ becomes infinite everywhere at the boundary of the region $D$ is that $D$ is a region of holomorphy.

On the other hand, we have simple examples of domains of holomorphy $H$ such that $\log K_{H}(z, \bar{z})$ does not become infinite everywhere at the boundary of $H$ (Bremermann [2]).

However, any domain of holomorphy is the limit of a monotonously increasing sequence of domains of holomorphy in which the function $\log K(\bar{z}, \bar{z})$ does become infinite everywhere at the boundary (Bremermann [2]).

7.6. In the case of one complex variable the "classical" Bergman kernel function $K_{D}(z, \bar{z})$ which is based on the inner product

$$
(f, g)=\int_{D} f \bar{g} d \omega
$$

remains finite $\left(K_{D}^{-1}(z, \bar{z})\right.$ greater than zero) at isolated boundary points, (Bremermann [2]).

We should like to mention: If we introduce an inner product with a certain 
"weighting function," we can obtain a kernel function that does become infinite everywhere at the boundary.

We introduce as inner product (let $D$ be bounded)

$$
(f, g)=\int_{D} f(z) \bar{g}(\bar{z}) \delta_{D}^{2}(z) d \omega_{z} .
$$

The whole kernel function theory works just the same (compare Nehari [1], Bergman [2]) as in the case of the inner product $\int_{D} f \bar{g} d \omega$.

Let the "reproducing kernel" (Aronszajn [1]) that belongs to $\int_{D} f \bar{g} \delta_{D}^{2}(z) d \omega$ be $k_{D}(z, \bar{z})$. Then, just as for $K_{D}(z, \bar{z})$ we have for $k_{D}(z, \bar{z})$ the representation

$$
\left(k_{D}\left(z^{(0)}, \bar{z}^{(0)}\right)\right)^{-1}=\min \int_{D}|f(z)|^{2} \delta_{D}^{2}(z) d \omega_{z},
$$

the minimum taken with respect to all functions $f(z)$ with $\left|f\left(z^{(0)}\right)\right| \geqq 1$.

Let $z^{(1)}$ be a boundary point of $D$. Then we take the function $f(z)$ $\equiv \epsilon /\left(z-z^{(1)}\right)$. We have by definition of the euclidean distance function:

$$
\delta_{D}(z) \leqq\left|z-z^{(1)}\right|
$$

Therefore

$$
\int_{D} \frac{\epsilon^{2}}{\left|z-z^{(1)}\right|^{2}} \delta_{D}^{2}(z) d \omega \leqq \epsilon^{2} \int_{D} d \omega=\epsilon^{2} \cdot \text { Area of } D<\infty .
$$

Therefore $\epsilon /\left(z-z^{(1)}\right)$ is (for any $\epsilon$ ) a function in our class (of functions for which $(f, f)<\infty)$. $\left[\epsilon /\left(z-z^{(1)}\right)\right.$ is in general not square integrable and therefore not in the class of functions considered in the case of the "classical" Bergman kernel function. That makes the difference. Compare Bremermann [2].]

For $\left|z-z^{(1)}\right|<\epsilon$ we have $|f(z)|=\epsilon /\left|z-z^{(1)}\right|>1$. Therefore we have

$$
\text { for }\left|z-z^{(1)}\right|<\epsilon:\left(k_{D}(z, \bar{z})\right)^{-1} \leqq \epsilon^{2} \text { (Area of } D \text { ). }
$$

That means obviously that $\dot{k_{D}}(z, \bar{z})$ goes to infinity everywhere at the boundary of $D$.

This method is limited to one variable.

\section{Pseudo-convex regions.}

8.1. We say that "the theorem of continuity" holds for a region $D$ with respect to the family of sets $\left\{S_{\nu}, T_{\nu}, S_{0}, T_{0}\right\}$ if the condition $S_{\nu}, T_{\nu}, T_{0} \subset \subset D, \lim _{\nu \rightarrow \infty} S_{\nu}$ $=S_{0}$ and $\lim _{\nu \rightarrow \infty} T_{\nu}=T_{0}$ implies $S_{0} \subset \subset D$.

8.2. Let $D$ be a region such that for a certain norm the function $-\log \Delta_{D}(z)$ is plurisubharmonic in $D$. Then the theorem of continuity holds for $D$ with respect to bounded domains $S_{\nu}, S_{0}$ on analytic surfaces and their boundaries $T_{\nu}, T_{0}$.

The analytic surfaces may be as defined in 4.2. The theorem follows exactly as Theorem 6.1 when we consider the fact that the maximum principle holds for domains on analytic surfaces and their boundaries with respect to 
the class of plurisubharmonic functions, as we have shown in 4.5.

8.3. Let the theorem of continuity hold for a region $D$ with respect to domains $S_{\nu}$ and their boundaries $T_{\nu}$ on analytic surfaces. Let $\Delta_{D}(z)$ and $\Delta_{a, D}(z)$ be distance functions based on an arbitrary norm. Let $\chi(\xi)$ be an arbitrary function that is holomorphic and different from zero in $D$. Then the minimum principle holds for the functions $|\chi(\xi)| \Delta_{D}(z)$ and $|\chi(\xi)| \Delta_{a, D}(z)$ with respect to domains $S \subset C D$ on analytic planes of one complex dimension and their boundaries $T \subset \subset D$.

In other words, our fundamental Theorem 5.1 (5.4) holds under the restriction that the sets $S, T$ are of a special form.

Proof. Let $S$ be a domain on the analytic plane $\left\{z \mid z=z^{(0)}+\lambda \mathfrak{b}\right\}$ where $\mathfrak{b}$ is a complex vector. Let $\bar{S} \cup \bar{T} \subset D$. Then we have

$$
\inf _{z \in T} \Delta_{a, D}(z)|\chi(z)|=m>0 .
$$

$\chi(z)$ being different from zero we have

$$
\Delta_{a, D}(z) \geqq m\left|\chi(z)^{-1}\right|
$$

for $z \in T$.

Then the points

$$
T(\tau)=\left\{z \mid z=z^{\prime}+\mathfrak{a}(m-\tau) \chi\left(z^{\prime}\right)^{-1} \wedge z^{\prime} \in T\right\}
$$

are contained in $D$ for any $m \geqq \tau>0$. For $\tau=m$ we have $T(\tau)=T$. Let us also consider the point set

$$
S(\tau)=\left\{z \mid z=z^{\prime}+\mathfrak{a}(m-\tau) \chi\left(z^{\prime}\right)^{-1} \wedge z^{\prime} \in S\right\} .
$$

$S(\tau)$ is a domain on an analytic surface. It has the parameter-representation

$z=z^{(0)}+\lambda \mathfrak{b}+\mathfrak{a}(m-\tau) \chi^{-1}\left(\mathfrak{z}^{(0)}+\lambda_{0}\right), \quad \lambda \in S^{*}, S^{*}$ a domain in the $\lambda$-plane.

It is

$$
\frac{\partial z_{j}}{\partial \lambda}=b_{j}+a_{j}(m-\tau)\left(\sum_{k=1}^{n} \frac{\partial \chi^{-1}\left(z^{0}+\lambda \mathfrak{b}\right)}{\partial z_{k}} b_{k}\right) .
$$

Now if the vectors $\mathfrak{a}$ and $\mathfrak{b}$ are linearly dependent, then the theorem is trivial. Let therefore the vectors $\mathfrak{a}$ and $\mathfrak{b}$ be linearly independent. Then $\partial z / \partial \lambda$ does not vanish. Therefore $S(\tau)$ is an analytic surface with no exceptional points. For such surfaces the theorem of continuity holds by assumption. Now for $\tau=m$ we have $T(\tau)=T$ and $S(\tau)=S$. There cannot exist a first $\tau_{0}>0$ such that $S\left(\tau_{0}\right)$ would not be contained in $D$. This would violate the theorem of continuity. Therefore we have

$$
S(\tau) \subset D \quad \text { for } m \geqq \tau>0 .
$$

From this we conclude

$$
\Delta_{a, D}(z) \geqq m|\chi(z)|^{-1} \quad \text { for } z \in S .
$$


From this follows

$$
\inf _{\mathfrak{z} \in S \cup T} \Delta_{\mathfrak{a}, D}(\mathfrak{z})|\chi(\mathfrak{z})|=\inf _{\mathfrak{z} \in T} \Delta_{\mathfrak{a}, D}(\mathfrak{z})|\chi(\mathfrak{z})|,
$$

and from this equality follows as in $\mathbf{5 . 1}$

$$
\inf _{z \in S \cup T} \Delta_{D}(z)|\chi(z)|=\inf _{z \in T} \Delta_{D}(z)|\chi(z)| . \quad \text { q.e.d. }
$$

8.4. If the theorem of continuity holds in the form as described in 8.2 then the functions $-\log \Delta_{a, D}(z)$ and $-\log \Delta_{D}(\xi)$ are plurisubharmonic in $D$.

This follows exactly as in 7.1. We observe that the maximum principle is actually used in 7.1 only for the special case that $S, T$ are domains and their boundaries on one-dimensional analytic surfaces. Considering 8.2 we obtain:

8.5. THEOREM. If the function $-\log \Delta_{D}(z)$ is plurisubharmonic in a region $D$ where $\Delta_{D}(z)$ is based upon one particular norm, then any function - log $\Delta_{D}^{\prime}(z)$ is plurisubharmonic, where $\Delta_{D}^{\prime}(z)$ is based upon an arbitrary norm.

REMARK. In particular we can choose the functions $-\log \delta_{D}(z)$ and $-\log \delta_{D}^{*}(z)$ where $\delta_{D}(z)$ is the euclidean distance function and $\delta_{D}^{*}(z)$ the distance function based on the maximum metric.

8.6. THEOREM. For an arbitrary norm the function $-\log \Delta_{D}(z)$ is plurisubharmonic in $D$ if and only if the theorem of continuity holds for domains $S_{\text {, }}$ and their boundaries $T$, on analytic surfaces of one complex dimension.

8.7. Definition. We term the regions for which the functions $-\log \Delta_{D}(\mathrm{z})$ are plurisubharmonic "pseudo-convex."

An equivalent definition is according to 8.4: A region is pseudo-convex if and only if the function - $\log \delta_{D}(z)$ is plurisubharmonic in $D$.

8.8. This definition brings Theorem 7.1 to the form: Any region of holomorphy is a pseudo-convex region.

The question arises if the converse is true, that is: Is any pseudo-convex region a region of holomorphy? This question (in a different, however essentially equivalent form) has been a problem since 1911. The problem was solved affirmatively in 1942 by K. Oka [1] for two complex dimensions and in 1953 and 1954 by K. Oka [2], F. Norguet [1] and H. J. Bremermann [4] for $n$ complex dimensions.

So far we have studied the pseudo-convex regions and the plurisubharmonic functions in connection with regions of holomorphy. In the following we shall investigate them for their own sake.

9. The formal correspondence of the plurisubharmonic functions to the convex functions and the pseudo-convex regions to the convex domains.

9.1. We should like to demonstrate that the pseudo-convex domains and the plurisubharmonic functions may be considered as the natural extension of the 
notion "convex domain" and "convex function" from the n-dimensional real space $R^{n}$ to the complex $n$-dimensional complex space $C^{n}$. We shall try to do this by matching corresponding theorems ${ }^{3}$ ). (Of course, one can consider convex domains and functions in the $2 n$-dimensional real space $R^{2 n}$ that is carrying the $C^{n}$ as a complex structure. However, doing this is something completely different.)

9.2. A real-valued function $U(x)$ of one real variable is called "convex" (or might be called "sublinear," compare T. Radó [1, p. 1]) in a domain $B$ of the real axis $R^{1}$ if and only if the following condition holds for any compact "subdomain" (subinterval) $G$ of $B$ :

If $l(x)$ is a linear function such that

$$
l(x) \geqq U(x)
$$

on the boundary of $G$, then

$$
l(x) \geqq U(x)
$$

holds throughout $G$.

9.2.* A real-valued function $V(z)$ of one complex variable is called "subharmonic" (compare T. Radó [1]) in a region (or domain) $D$ of the plane of one complex variable $C^{1}$, if and only if $-\infty \leqq V(z)<\infty$ and $V(z)$ is upper semi-continuous in $D$ and the following condition holds for any compact subdomain $G$ of $D$ :

If $h(z)$ is a function, harmonic in $G$ and continuous in $G^{*}$ such that $h(z) \geqq V(z)$ on the boundary of $G$, then

$$
h(z) \geqq V(z)
$$

holds throughout $G$.

9.3. A real-valued function $U(\mathfrak{x})$ of $n$ real variables $\mathfrak{x}=\left(x_{1}, \cdots, x_{n}\right)$ is called "convex" (or might be called "plurisublinear") in a domain $B$ of the space of $n$ real variables $R^{n}$ if and only if on every straight line $\mathfrak{x}=\mathfrak{x}^{(0)}+t \mathfrak{b}$ ( $t$ a real parameter, $\mathfrak{b}$ a real vector) it is a convex function of the parameter $t$ in the intersection $B \cap\left\{\mathfrak{x} \mid \mathfrak{x}=\mathfrak{x}^{(0)}+t \mathfrak{b}\right\}$ (Bonnesen-Fenchel [1], Bourbaki $[1])$.

9.3.* A real-valued function $V(z)$ of $n$ complex variables $z=\left(z_{1}, \cdots, z_{n}\right)$ is called "plurisubharmonic" (or "pseudo-convex" by $\mathrm{K}$. Oka [1] and in previous papers of the author, Bremermann $[1 ; 2])$ in a region $D$ of the space of $n$ complex variables $C^{n}$ if and only if $-\infty \leqq V(z)<\infty$ and $V(z)$ is upper semi-continuous in $D$ and on every analytic plane $z=z^{(0)}+\lambda \mathfrak{a}$ (a a complex vector) it is a subharmonic function of the parameter $\lambda$ in the intersection $D \cap\left\{z \mid z=z^{(0)}+\lambda a\right\}$. (See 2.6.)

(3) The proofs for the theorems in the convex case are either trivial or can be found in Bourbaki [1], in Bonnesen-Fenchel [1], or follow from 11.4. 
9.4. $U(x)$ is convex in $B$ if and only if

$$
d^{2} U / d x^{2} \geqq 0
$$

at every point in $B$ (Bonnesen-Fenchel $[1$, p. 18]). (The differentiation in the sense of Schwartz [1].)

$U(x)$ is linear if and only if

$$
d^{2} U / d x^{2}=0 .
$$

9.4. ${ }^{*} V(z)$ is subharmonic in $D$ if and only if

$$
\partial^{2} V / \partial z \partial \bar{z} \geqq 0
$$

at every point in $D$ (Radó $[1, \mathrm{p} .12]$ ). (The differentiation in the sense of Schwartz.)

$V(z)$ is harmonic if and only if

$$
\partial^{2} V / \partial z \partial \bar{z}=0 .
$$

9.5. $U(\mathfrak{x})$ is convex in $B$ if and only if the quadratic form

$$
\sum_{\mu, v=1}^{n} \frac{\partial^{2} U}{\partial x_{\mu} \partial x_{\nu}} d x_{\mu} d x_{\nu}
$$

is positive semidefinite at every point of $B$. (The differentiation in the sense of Schwartz.) (Bonnesen-Fenchel [1, p. 18]).

$U(\mathfrak{x})$ is linear in $B$ if and only if

$$
\sum_{\mu, \nu=1}^{n} \frac{\partial^{2} U}{\partial x_{\mu} \partial x_{\nu}} d x_{\mu} d x_{\nu}=0
$$

in $B$.

9.5.* $V(z)$ is plsh. in $D$ if and only if the Hermitian form

$$
\sum_{\mu, v=1}^{n} \frac{\partial^{2} V}{\partial z_{\mu} \partial \bar{z}_{\nu}} d z_{\mu} d \bar{z}_{\nu}
$$

is positive semidefinite at every point of $D$. (The differentiation in the sense of Schwartz.) (Bremermann [1, p. 21], Lelong [2, p. 194].)

$V(z)$ is pluriharmonic (that means $V(\mathfrak{z})$ is the real part of a holomorphic function) if and only if

$$
\sum_{\mu, \nu=1}^{n} \frac{\partial^{2} V}{\partial z_{\mu} \partial \bar{z}_{\nu}} d z_{\mu} d \bar{z}_{\nu}=0
$$

9.6. A convex function assumes its maximum at the boundary of a domain unless it is constant in the domain.

9.6. A plsh. function takes its maximum at the boundary of a domain unless it is a constant in the domain (Bremermann [1], also Lelong [1]). 
9.7. If $U(\mathfrak{x})$ is convex in $B$ and $\left\{\mathfrak{x} \mid \mathfrak{x}=\mathfrak{x}^{(0)}+t \mathfrak{b}\right\} \subset \subset B$ for $|t| \leqq r$, then

$$
U\left(\mathfrak{x}^{(0)}\right) \leqq \frac{U\left(\mathfrak{x}^{(0)}+\mathfrak{r b}\right)-U\left(\mathfrak{x}^{(0)}-\mathfrak{r b}\right)}{2} .
$$

If for any $\mathfrak{x}^{(0)} \in B$ there exists an $r_{0}$ such that for all $r \leqq r_{0}$ and for all $\mathfrak{b}$ with $|\mathfrak{b}|=1$

$$
U\left(\mathfrak{x}^{(0)}\right) \leqq \frac{U\left(\mathfrak{x}^{(0)}+r \mathfrak{b}\right)-U\left(\mathfrak{k}^{(0)}-r \mathfrak{b}\right)}{2},
$$

then $U(\mathfrak{x})$ is convex in $B$.

9.7.* If $V(z)$ is plsh. in $D$ and if $\left\{z \mid z=z^{(0)}+\lambda a\right\} \subset \subset D$ for $|\lambda| \leqq r$, then

$$
V\left(\mathfrak{z}^{(0)}\right) \leqq \frac{1}{2 \pi} \int_{0}^{2 \pi} V\left(\mathfrak{z}^{(0)}+r e^{i \phi} \mathfrak{a}\right) d \phi .
$$

Let $-\infty \leqq V(z)<\infty$ and let $V(z)$ be upper semicontinuous. Then $V(z)$ is plsh. in $D$, if for any $z^{(0)} \in D$ there exists an $r_{0}$ such that for all $r \leqq r_{0}$ and for all $a$ with $|\mathfrak{a}|=1$

$$
V\left(\mathfrak{z}^{(0)}\right) \leqq \frac{1}{2 \pi} \int_{0}^{2 \pi} V\left(\mathfrak{z}^{(0)}+r e^{i \phi} \mathfrak{a}\right) d \phi
$$

(Bremermann [1, p. 22], also Lelong [1]).

9.8. Let $C\left(\mathfrak{x}^{(0)}, r\right)=\left\{\mathfrak{x}|| x_{1}-x_{1}^{(0)}|<r \wedge \cdots \wedge| x_{n}-x_{n}^{(0)} \mid<r\right\}$. Then, if $U(\mathfrak{x})$ is convex in $B$ and $C\left(\mathfrak{x}^{(0)}, r\right) \subset \subset B$, then

$$
U\left(\mathfrak{x}^{(0)}\right) \leqq \frac{1}{(2 r)^{n}} \int_{C\left(\mathfrak{r}^{(0)}, r\right)} U(\mathfrak{x}) d x_{1} \cdots d x_{n} .
$$

9.8.* Let $C\left(z^{(0)}, r\right)=\left\{z|| z_{1}-z_{1}^{(0)}|<r \wedge \cdots \wedge| z_{n}-z_{n}^{(0)} \mid<r\right\}$. Then if $V(z)$ is plsh. in $D$ and $C\left(z^{(0)}, r\right) \subset \subset D$, then

$$
V\left(\mathfrak{z}^{(0)}\right) \leqq \frac{1}{\left(\pi r^{2}\right)^{n}} \int_{C\left(z^{(0)}, r\right)} V(\mathfrak{z}) d x_{1} d y_{1} \cdots d x_{n} d y_{n}
$$

(Bremermann [1, p. 24], also Lelong [1]).

9.9. Theorem 9.6 holds if we replace the $n$-cube $C\left(\mathfrak{x}^{(0)}, r\right)$ by the $n$-sphere $\{\mathfrak{x}|| \mathfrak{x} \mid<r\}$ and the volume of the $n$-cube, $(2 r)^{n}$, by the volume of the $n$ sphere.

9.9.* Theorem 9.6 holds if we replace the polycylinder $C\left(z^{(0)}, r\right)$ by the $2 n$-sphere $\{z|| z \mid<r\}$ and the volume of the polycylinder, $\left(\pi r^{2}\right)^{n}$, by the volume of the $2 n$-sphere.

9.10. A function is convex in a domain $B$ if and only if it is convex in the neighborhood of each of its points. 
9.10.* A function is plsh. in a region $D$ if and only if it is plsh. in the neighborhood of each of its points (consequence of 9.7).

9.11. If $U_{1}(\mathfrak{x})$ and $U_{2}(\mathfrak{x})$ are convex in $B$, then $U_{1}(\mathfrak{x})+U_{2}(\mathfrak{x})$ is convex in $B$. If $U(\mathfrak{x})$ is convex in $B$, then $\alpha U(\mathfrak{x})$ is convex in $B$ if $\alpha \geqq 0$. If $U(\mathfrak{x})$ is convex in $B$ and non-negative, then $U^{2}(\mathfrak{x})$ is convex in $B$. If $U(\mathfrak{x})$ is convex in $B$, then $e^{U(\mathfrak{F})}$ is convex in $B$.

9.11.* If $V_{1}(z)$ and $V_{2}(z)$ are plsh. in $D$, then $V_{1}(z)+V_{2}(z)$ is plsh. in $D$. If $V(z)$ is plsh. in $D$, then $\alpha V(z)$ is plsh. in $D$ if $\alpha \geqq 0$. If $V(z)$ is plsh. in $B$ and non-negative, then $V^{2}(z)$ is plsh. in $D$. If $V(z)$ is plsh. in $D$, then $e^{V(z)}$ is plsh. in $D$ (Bremermann [1], also Lelong [1]).

9.12. The upper envelope $U(\mathfrak{x})=\lim \sup _{\mathfrak{x}^{\prime} \rightarrow \mathfrak{r}} \sup _{\alpha}\left\{U_{\alpha}\left(\mathfrak{x}^{\prime}\right)\right\}$ of a family of functions $\left\{U_{\alpha}\right\}$ that are convex in a domain $B$ and bounded in every closed subdomain of $B$ is a convex function.

9.12.* The upper envelope $V(z)=\lim \sup _{z^{\prime} \rightarrow z} \sup _{\alpha}\left\{V_{\alpha}\left(z^{\prime}\right)\right\}$ of a family of functions $\left\{V_{\alpha}(\xi)\right\}$ that are plurisubharmonic in a region $D$ and bounded in every closed subregion of $D$ is a plsh. function (Bremermann [1]).

9.13. The limit of a uniformly convergent sequence of convex functions is a convex function.

9.13.* The limit of a uniformly convergent sequence of plsh. functions is a plsh. function (Bremermann [1, p. 30], also Lelong [1]).

9.14. Any function $U(\mathfrak{x})$ that is convex in a domain $B$ can be approximated in any closed subdomain $B^{\prime} \subset \subset B$ by a sequence of in $B^{\prime} k$ times ( $k$ an arbitrary integer) differentiable convex functions $U_{\nu}(\mathfrak{x})$ such that

$$
U_{\nu}(\mathfrak{x}) \geqq U_{\nu+1}(\mathfrak{x}) \geqq U(\mathfrak{x}) \text { in } B^{\prime}
$$

and

$$
\lim _{\nu \rightarrow \infty} U_{\boldsymbol{\nu}}(\mathfrak{x})=U(\mathfrak{x}) .
$$

9.14.* Any function $V(z)$ that is plsh. in a region $D$ can be approximated in any closed subregion $D^{\prime} \subset \subset D$ by a sequence of in $D^{\prime} k$ times $(k$ an arbitrary integer) differentiable plsh. functions $V_{\nu}(z)$ such that

$$
V_{v}(z) \geqq V_{v+1}(z) \geqq V(z) \text { in } D^{\prime}
$$

and

$$
\lim _{\nu \rightarrow \infty} V_{\nu}(z)=V(z)
$$

(Bremermann [1, p. 30]).

9.15. This list could be continued rather indefinitely. By substituting "linear" for "harmonic," "straight line" for "analytic plane," x for z, etc., we obtain corresponding theorems for plurisubharmonic and convex functions.

In the following paragraphs we shall match corresponding theorems for pseudo-convex regions and convex domains. The pseudo-convex regions are 
such that $-\log \Delta_{D}(\mathrm{z})$ is a plurisubharmonic function in $D$. However, it is sufficient to consider only the euclidean norm to characterize the pseudoconvex regions: A region is pseudo-convex if and only if $-\log \delta_{D}(z)$ is plurisubharmonic in $D(8.6)$. The key for establishing the correspondence between the convex domains and the pseudo-convex regions is that we are able to prove that a domain $B C R^{n}$ is convex if and only if $-\log \delta_{B}(\mathfrak{x})$ is a convex function in $B . \delta_{B}(\mathfrak{x})$ is the euclidean distance function (compare 3.4). We limit ourselves here for simplicity's sake to the euclidean norm. It should be noted that one can prove: If a domain $B$ is convex, then $-\log \Delta_{B}(\mathfrak{x})$ is a convex function in $B$, where $\Delta_{B}(\mathfrak{x})$ is based upon an arbitrary norm of the $R^{n}$, and conversely if $-\log \Delta_{B}(\mathfrak{x})$ is a convex function in $B$, where $\Delta_{B}(\mathfrak{x})$ is based on an arbitrary norm, then $B$ is a convex domain. The proof follows similar lines as the proof for the fact that if the theorem of continuity holds for a region $D \subset C^{n}$, then $-\log \Delta_{D}(z)$ is plurisubharmonic and vice versa. The proof that $-\log \delta_{B}(\mathfrak{x})$ is convex in a convex domain, which we will give in the following, is different.

9.16. A domain $B \subset R^{n}$ is convex if and only if $-\log \delta_{B}(\mathfrak{x})$ is a convex function in $B$.

A convex domain is a domain such that with two points the straight line segment connecting the two points belongs to the domain. A domain is convex if and only if through every boundary point we have a supporting linear manifold of dimension $n-1$ (where $n$ is the dimension of the domain). For definition etc. see: Bourbaki [1], Behnke-Stein [2], Bonnesen-Fenchel [1]. We shall first prove part of Theorem 9.16:

9.17. If $B$ is a convex domain, then $-\log \delta_{B}(\mathfrak{x})$ is a convex function in $B$.

Proof. Through every boundary point $\mathfrak{x}^{(0)}$ of a convex domain we have a supporting plane (or hyperplane) which can be written in the form $\{\mathfrak{x} \mid \mathfrak{a} \cdot$ (x $\left.\left.-\mathfrak{x}^{(0)}\right)=0\right\}$ where $\mathfrak{a}$ is a real vector, orthogonal to the plane; $\mathfrak{a} \cdot\left(\mathfrak{x}-\mathfrak{x}^{(0)}\right)$ denotes the scalar product of $\mathfrak{a}$ and $\mathfrak{x}-\mathfrak{r}^{(0)}$.

Let $|\mathfrak{a}|=1$ and $\mathfrak{a}$ such that $\mathfrak{a} \cdot\left(\mathfrak{x}-\mathfrak{x}^{(0)}\right)$ is positive on that side of the plane on which $B$ is situated. Then $\mathfrak{a} \cdot\left(\mathfrak{x}-\mathfrak{x}^{(0)}\right)$ measures in $B$ the distance of the point $\mathfrak{x}$ from the plane. Obviously

$$
\delta_{B}(\mathfrak{x})=\min \left\{\mathfrak{a} \cdot\left(\mathfrak{x}-\mathfrak{x}^{(b)}\right)\right\}
$$

where $\mathfrak{x}^{(b)}$ varies through all boundary points of $B$ and $\mathfrak{a}$ is the vector associated to $\mathfrak{x}^{(b)}$ as described above. Then

$$
-\log \delta_{B}(\mathfrak{x})=\max \left\{-\log \mathfrak{a} \cdot\left(\mathfrak{x}-\mathfrak{x}^{(b)}\right)\right\} .
$$

Each function $-\log \mathfrak{a} \cdot\left(\mathfrak{x}-\mathfrak{x}^{(b)}\right)$ is a convex function in $B$, because

$$
\sum_{\mu, \nu=1}^{n} \frac{\partial^{2}\left(-\log \mathfrak{a} \cdot\left(\mathfrak{x}-\mathfrak{x}^{(b)}\right)\right.}{\partial x_{\mu} \partial x_{\nu}} d x_{\mu} d x_{\nu}=\sum_{\mu, \nu=1}^{n} \frac{a_{\mu} a_{\nu} d x_{\mu} d x_{\nu}}{\left(\mathfrak{a} \cdot\left(\mathfrak{x}-\mathfrak{x}^{(b)}\right)\right)^{2}}=\frac{(\mathfrak{a} \cdot d \mathfrak{r})^{2}}{\left(\mathfrak{a} \cdot\left(\mathfrak{x}-\mathfrak{x}^{(b)}\right)\right)^{2}} \geqq 0
$$

(compare 9.5). Also the family of functions $\left\{-\log \mathfrak{a} \cdot\left(\mathfrak{x}-\mathfrak{x}^{(b)}\right)\right\}$ is bounded 
in any closed subdomain $B^{\prime} \subset \subset B$. Therefore, according to 9.12 , the upper envelope of the family, and that is $-\log \delta_{B}(\mathfrak{x})$, is a convex function in $B$.

Thus we have proved, if $B$ is convex, then $-\log \delta_{B}(\mathfrak{x})$ is convex in $B$.

9.18. Let $-\log \delta_{B}(\mathfrak{r})$ be a convex function in $B$. Then the following "theorem of continuity" holds for $B$ :

Let $S_{\nu}$ and $S_{0}$ be straight line segments and $T_{\nu}$ and $T_{0}$, respectively, the boundary (end points) of $S_{\nu}$ and $S_{0}$, respectively. Let $S_{\nu} \cup T_{\nu} \subset B$ for every $\nu$ and let $\lim _{\nu \rightarrow \infty} S_{\nu}=S_{0}$ and $\lim _{\nu \rightarrow \infty} T_{\nu}=T_{0}$. Then, if $T_{0} \subset B, S_{0} \subset B$.

Proof. Because of the maximum principle (9.6), we have for every $\nu$

$$
\max _{\mathfrak{r} \in S_{\nu} \cup T_{\nu}}-\log \delta_{B}(\mathfrak{x})=\max _{\mathfrak{r} \in T_{\nu}}-\log \delta_{B}(\mathfrak{x}),
$$

which is equivalent to

$$
\min _{\mathfrak{x} \in S_{\nu} \cup T_{\nu}} \delta_{B}(\mathfrak{x})=\min _{\mathfrak{x} \in T_{\nu}} \delta_{B}(\mathfrak{x}) .
$$

Now $\delta_{B}(\mathfrak{x})$ is continuous in $B$ (compare 3.2). Therefore, the latter inequality also holds in the limit:

$$
\min _{\mathfrak{x} \in S_{0} \cup T_{0}} \delta_{B}(\mathfrak{x})=\min _{\mathfrak{x} \in T_{0}} \delta_{B}(\mathfrak{x}) .
$$

If $T_{0} \in B$, then $\min _{\mathfrak{x} \in T_{0}} \delta_{B}(\mathfrak{x})=m>0$. Therefore

$$
\min _{\mathfrak{x} \in S_{0} \cup T_{0}} \delta_{B}(\mathfrak{x})=m>0 .
$$

It follows that $S_{0} \cup T_{0} \subset B$.

9.19. If the "theorem of continuity" (9.18) holds for a domain $B$, then $B$ is a convex domain.

Proof. Let $\mathfrak{x}^{(1)}$ and $\mathfrak{x}^{(2)}$ be two points in $B$. We connect $\mathfrak{x}^{(1)}$ and $\mathfrak{x}^{(2)}$ by a curve $\{\mathfrak{x} \mid \mathfrak{x}=\mathfrak{x}(t), 0 \leqq t \leqq 1\}$ in $B$. Let $\mathfrak{x}(0)=\mathfrak{x}^{(1)}$ and $\mathfrak{x}(1)=\mathfrak{r}^{(2)}$. If $t$ is close to zero, then the line segment from $\mathfrak{r}^{(1)}$ to $\mathfrak{x}(t)$ is contained in $B$, as $\mathfrak{x}^{(1)}$ is an interior point of $B$.

If then $t$ increases toward 1 , there cannot be a first $t_{0}$ such that the segment from $\mathfrak{x}^{(0)}$ to $\mathfrak{x}(t)$ is contained in $B$ for $1 \leqq t<t_{0}$, but not for $t_{0}$, because that would be a contradiction to our theorem of continuity. Therefore, the segment $\mathfrak{x}(0)$ to $\mathfrak{x}(1)$ which is the segment from $\mathfrak{x}^{(1)}$ to $\mathfrak{x}^{(2)}$ belongs to $B$.

Thus we have proved: For arbitrary points $\mathfrak{x}^{(1)}$ and $\mathfrak{x}^{(2)} \in B$, the points on the connecting straight line are contained in $B$. That means $B$ is a convex domain.

Theorems $9.17,9.18$, and 9.19 together yield Theorem 9.16.

9.20. $A$ domain $B$ is convex if and only if $-\log \delta_{B}(\mathfrak{x})$ is a convex function in $B$ (Theorem 9.16).

9.20.* A region $D$ is pseudo-convex if and only if $-\log \delta_{D}(z)$ is a plsh. function in $D$ (Definition 8.1). 
9.21. The (open) intersection $B$ of a family of convex domains $\left\{B_{\alpha}\right\}$ is a convex domain.

Proof. We consider the theorem to be true if the open intersection is empty. If it is not empty, then the family of the distance functions

$$
\left\{-\log \delta_{B_{\alpha}}(\mathfrak{x})\right\}
$$

is uniformly bounded in every closed subdomain of $B .-\log \delta_{B}(\mathfrak{x})$ is the upper envelope of the family $\left\{-\log \delta_{B_{\alpha}}(\mathfrak{x})\right\}$. Each $-\log \delta_{B_{\alpha}}(\mathfrak{x})$ is a convex function in $B_{\alpha}$ and therefore also in $B$. The upper envelope of a family of convex functions is convex (9.12). Therefore, $-\log \delta_{B}(\mathfrak{x})$ is convex in $B$ which implies (9.20) that $B$ is a convex region.

9.21.* The (open) intersection $D$ of a family of pseudo-convex regions $\left\{D_{\alpha}\right\}$ is a pseudo-convex region.

REMARK. In the convex case the intersection of convex domains is again a domain. In the pseudo-convex case the intersection of pseudo-convex domains can be a region. We see that immediately if we remember that in the $z$-plane any domain is a pseudo-convex domain.

The intersection of regions, however, is again a region.

The proof of our theorem for the pseudo-convex case goes exactly as in the convex case.

9.22. A domain $B$ is convex if and only if it satisfies the "theorem of continuity" (as described in 9.18).

In 9.17 we have proved: If $B$ is convex, then $-\log \delta_{B}(\mathfrak{x})$ is convex in $B$. In 9.18: If $-\log \delta_{B}(\mathfrak{x})$ is convex in $B$, then the theorem of continuity holds. In 9.19: If the theorem of continuity holds, then $B$ is convex.

9.22.* $A$ region $D$ is pseudo-convex if and only if it satisfies the following "theorem of continuity": Let $S_{\nu}$ be domains on 2-dimensional analytic surfaces, $T_{\nu}$ the boundaries. Let $\lim _{\nu \rightarrow \infty} S_{\nu}=S_{0}$ and $\lim _{\nu \rightarrow \infty} T_{\nu}=T_{0}$. Let $S_{\nu} \cup T_{\nu} \subset \subset B$ and $T_{0} \subset \subset B$, and let $S_{0} \cup T_{0}$ be bounded. Then $S_{0} \subset \subset B$ (Theorem 8.6).

9.23. If $U(\mathfrak{x})$ is a convex function in a domain $B$ and if (for real $M$ ) the domain

$$
\{\mathfrak{x} \mid \mathfrak{x} \in B \wedge U(\mathfrak{x})<M\}
$$

is compact in $B$, then it is a convex domain.

One sees easily that the point set

$$
\{\mathfrak{x} \mid \mathfrak{x} \in B \wedge U(\mathfrak{x})<M\}
$$

is a domain and that it satisfies the theorem of continuity because the maximum principle holds for the straight line segments $S_{\nu}, T_{\nu}$, and $U(\mathfrak{x})$ (compare 9.6). Therefore the region

$$
\{\mathfrak{x} \mid \mathfrak{x} \in B \wedge U(\mathfrak{x})<M\}
$$

is convex according to 9.22 . 
9.23. * If $V(z)$ is a plsh. function in a region $D$ and if (for real $M$ ) the region

$$
\{z \mid z \in D \wedge V(z)<M\}
$$

is compact in $D$, then it is a pseudo-convex region ( $\left.{ }^{4}\right)$.

One sees easily that the region

$$
\{z \mid z \in D \wedge V(z)<M\}
$$

satisfies the theorem of continuity because the maximum principle holds for domains on analytic surfaces and their boundaries, $S_{\nu}, T_{\nu}$, and $V(z)$ (compare 4.6). Therefore the region

$$
\{z \mid z \in D \wedge V(z)<M\}
$$

is pseudo-convex according to 9.22.

9.24. Let $\left\{B_{v}\right\}$ be a sequence of convex domains, such that $B_{\nu} \subset B_{\nu+1} \subset B$, and $\lim _{\nu \rightarrow \infty} B_{\nu}=B_{0}$. Then $B$ is a convex domain $\left(^{5}\right)$.

9.24.* Let $\left\{D_{\nu}\right\}$ be a sequence of pseudo-convex regions, such that $D, \subset D_{\nu+1}$ $C D$, and $\lim _{\nu \rightarrow \infty} D_{\nu}=D$. Then $D$ is a pseudo-convex region $\left(^{6}\right)$.

Proof. Because of $B, \subset B_{v+1} \subset B$ we have $\delta_{B_{y}}(\mathfrak{x}) \leqq \delta_{B_{v+1}}(\mathfrak{x}) \leqq \delta_{B}(\mathfrak{x})$ which is equivalent to $-\log \delta_{B_{y}}(\mathfrak{x}) \geqq-\log \delta_{B_{\mathfrak{y}+1}}(\mathfrak{x}) \geqq-\log \delta_{B}(\mathfrak{x})$. In any compact subregion of $B$ the functions $-\log \delta_{B_{\nu}}(\mathfrak{x})$ converge uniformly towards $-\log \delta_{B}(\mathfrak{x})$. Every function $-\log \delta_{B_{y}}(\mathfrak{x})$ is convex because of 9.20 . Therefore $-\log \delta_{B}(\mathfrak{x})$ is convex because of 9.13 , and because of 9.20 it follows that $B$ is convex.

The proof for the pseudo-convex case is completely analogous to the convex case.

9.25. If $B$ is a domain such that there exists a function $U(\mathfrak{x})$ that is convex in $B$ and such that for arbitrary large real $M$

$$
\{\mathfrak{x} \mid \mathfrak{x} \in B \wedge U(\mathfrak{x})<M\} \subset \subset B,
$$

then $B$ is convex.

9.25.* If $D$ is a region such that there exists a function $V(z)$ that is plsh. in $D$ and such that for arbitrary large real $M$

$$
\{z \mid z \in D \wedge V(z)<M\} \subset \subset D
$$

then $D$ is pseudo-convex.

(4) The regions $\{z \mid z \in D \wedge V(z)<M\}$ are of considerable interest (compare Theorem 7.2, compare also Bremermann[1] and [2]).

Also, Marston Morse's theory of "critical points" (Marston Morse [1]) could be developed to a certain extent for these regions. The "subharmonic" and "plurisubharmonic functions" are a similar extension of the class of harmonic functions as the "pseudo-harmonic functions" of Marston Morse. The surfaces $V(z)=M$ correspond to the "level curves," and a "critical point" is a point, where $\partial V / \partial z_{1}=\cdots=\partial V / \partial z_{n}=0$ and $\partial V / \partial \bar{z}_{1}=\cdots=\partial V / \partial \bar{z}_{n}=0$ (if $V(\xi)$ is differentiable).

(b) For the definition of $\lim _{\nu \rightarrow \infty} B_{\nu}=B$ and $\lim _{\nu \rightarrow \infty} D_{\nu}=D$, see 2.4 .

(8) This theorem stated for regions of holomorphy is known as the "theorem of BehnkeStein" (compare 2.9). 
According to 9.23 every domain $B_{M}=\{\mathfrak{x} \mid \mathfrak{x} \in B \wedge U(\mathfrak{x})<M\}$ is convex. Also $B_{M} \subset B_{M+1} \subset B$ and $\lim _{M \rightarrow \infty} B_{M}=B$. Therefore $B$ is convex according to 9.24. The same argument proves also the pseudo-convex case.

We note that this theorem is important for 7.2.

9.26. If $B$ is a convex domain, then it can be approximated by convex domains $B$, such that the boundary is $k$ times ( $k$ an arbitrary integer) differentiable, and $B_{\nu} \subset B_{\nu+1} \subset B$ and $\lim _{\nu \rightarrow \infty} B_{\nu}=B$.

9.26.* If $D$ is a pseudo-convex region, then it can be approximated by pseudoconvex regions $D$, such that the boundary is $k$ times ( $k$ an arbitrary integer) differentiable, and $D_{v} \subset D_{v+1} \subset D$ and $\lim _{v \rightarrow \infty} D_{v}=D$.

Proof. (For the pseudo-convex case, the proof for the convex case is analogous). If $D$ is pseudo-convex, then $-\log \delta_{D}(z)$ is plsh. in $D$. In a (sufficiently large) subregion of $D$ we approximate $-\log \delta_{D}(z)$ by $k$-times continuous differentiable plsh. functions $V_{\boldsymbol{p}}(\mathfrak{z})$ and define

$$
D_{1}=\left\{z \in D \wedge V_{\nu_{1}}(z)<M_{1}\right\},
$$

$\nu_{1}$ and $M_{1}$ sufficiently chosen. The boundary of $D_{1}$ is given by $V_{\nu_{1}}(z)-M_{1}=0$ and is $k$-times differentiable. In a similar way we construct $D_{2}, D_{3}$ such that $D_{1} \subset D_{2} \subset D_{3}$, etc., and $\lim _{v \rightarrow \infty} D_{v}=D$.

This list could be continued further.

10. Pseudo-convex envelopes and the problem of complex manifolds and Riemann domains.

10.1. Any arbitrary domain $B C R^{n}$ has a convex envelope. The convex envelope is the intersection of all convex domains containing $B$.

Therefore we are tempted to define the pseudo-convex envelope of an arbitrary domain $D \subset C^{n}$ to be the intersection of all pseudo-convex domains containing $D$. This is possible, of course.

However, if we want the pseudo-convex envelope of a domain to coincide with the envelope of holomorphy, then this definition does not work. There are schlicht domains, the envelope of holomorphy of which is no longer schlicht (Thullen [1], Bremermann [3]). This leads us to consider pseudoconvex domains and plsh. functions in non-schlicht domains.

Complex manifolds have been studied extensively in recent years. Therefore, the extension of our techniques is of interest not only in connection with the pseudo-convex envelope.

10.2. A $2 n$-dimensional complex manifold $\mathcal{X}$ is a $2 n$-dimensional manifold that has the following property: $\mathcal{X}$ is covered with local systems of complex coordinates $P(z)$ (let $P$ denote a variable point in $\mathscr{X}$ ), such that in the intersection of two systems $P(z)$ and $P\left(z^{*}\right)$ the transformations $z^{*}\left(z^{*}\right)$ and $z^{*}(z)$ are holomorphic and one to one.

10.3. A function $V(P)$ is called plurisubharmonic in a region on a complex 
manifold $\mathcal{X}$ if $V(P)$ is upper semicontinuous and $V(P(z))$ is plsh. (in z) where the local coordinates $P(z)$ are valid.

Change of local coordinates does not affect the property of $V(P)$ to be plsh., as the property of a function to be plsh. is invariant with respect to holomorphic transformations.

If $\mathcal{X}$ is schlicht the definition coincides with our previous definition for schlicht domains.

10.4. A domain $D \subset C^{n}$ is pseudo-convex if and only if there exists a function $V(z)$ plsh. in $D$, such that for all (arbitrarily large) real $M$ the region

$$
\{z \mid z \in D \wedge V(z)<M\}
$$

is compact in $D$ (7.2 and 9.25).

Because this property is necessary and sufficient we can take it as definition; this definition can be carried over to complex manifolds.

$A$ region $D \subset \mathcal{X}, \mathfrak{X}$ a complex manifold, is called pseudo-convex if and only if there exists a function $V(P)$, plsh. in $D$, such that for all (arbitrary large) real $M$

$$
\{P \mid P \in \mathcal{H} \wedge V(P)<M\} \subset \subset D .
$$

10.5. This definition permits us to extend several of the theorems in $\S 9$ to complex manifolds.

The problem is, however, to show that domains of holomorphy on complex manifolds are pseudo-convex domains. This problem would be solved if it were possible to find a substitute for $-\log \delta_{D}(z)$ on complex manifolds.

Recently K. Oka has solved this problem for concrete complex manifolds over the $C^{n}$, with no branchpoints as interior points, that is complex manifolds such that the $z_{1} \cdots z_{n} \in C^{n}$ are local coordinates at every point of the manifold. On such a manifold $\delta_{D}(z)$ can be defined. The difficulty here is to show that $-\log \delta_{D}(z)$ goes to infinity also if $z$ moves on a path that is winding on infinitely many sheets such that the projection into the $C^{n}$ remains within a bounded domain (K. Oka [2]).

10.6. We note, in the case of regions $D$ that are compact in a Stein manifold (that is a special type of complex manifold), that we can imbed the non-schlicht region as an analytic surface in a higher dimensional schlicht space. In this case we can take a distance function in this higher-dimensional schlicht space, restrict it to $D$, and thus obtain a substitute for $-\log \delta_{D}(z)$.

10.7. The pseudo-convex envelope. In 10.1 we have seen that we have to consider not only schlicht pseudo-convex regions containing a given region in order to define the pseudo-convex envelope but also the non-schlicht regions containing the given region.

$B y$ "continuation" of a given schlicht region $D$ we shall understand any concrete complex manifold with no branchpoints as interior points that contains the given region $D$. 
As "pseudo-convex envelope" of a schlicht region we define the largest (open) region contained in the intersection of all continuations of $D$.

By this definition any region $D C C^{n}$ possesses a pseudo-convex envelope and it coincides, according to the result of $\mathrm{K}$. Oka, with the envelope of holomorphy of $D$.

10.8. Convex domains have the property that with two points the connecting straight line segment belongs to the domain. What corresponds in the pseudo-convex case to this property?

The straight line segment is the convex envelope of the set consisting of the two points. A domain $B$ is convex if and only if with any subset that is contained in the domain the convex envelope of the subset is contained in the domain. Thus it turns out that it is sufficient to take as subsets pairs of points of $B$ and to require that their convex envelope is contained in $B$.

Pseudo-convex regions also have the property that a region $D$ is pseudoconvex if with any subset its pseudo-convex envelope is contained in $D$. The question is: What kind of subsets are already characteristic and thus correspond to the pairs of points in the convex case? In other words we have the problem of determining a class $\mathbb{E}$ of sets as simple as possible such that a region $D$ is pseudo-convex if the pseudo-convex envelope of any subset of $D$ that belongs to $\mathbb{E}$ is contained in $D$.

11. Tube domains.

11.1. Let $z_{j}=x_{j}+i y_{j}$ and $\mathfrak{x}=\left(x_{1}, \cdots, x_{n}\right), \mathfrak{y}=\left(y_{1}, \cdots, y_{n}\right)$, and $z=\mathfrak{r}+i \mathfrak{\eta}$. $A$ "tube domain" is a domain that has the form

$$
T=\{z \mid \mathfrak{x} \in B, \mathfrak{y} \text { arbitrary }\},
$$

where the "base" $B$ is a domain in the space of the $n$ real parts $x_{1}, \cdots, x_{n}$.

We write $T_{B}$ for a tube with base $B$.

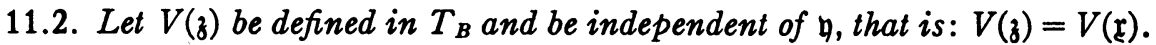
Then $V(\mathfrak{z})$ is plsh. in $T_{B}$ if and only if $V(\mathfrak{x})$ is convex in $B\left({ }^{7}\right)$.

Proof. $V(z)$ is pseudo-convex if and only if

$$
\sum_{\mu, v=1}^{n} \frac{\partial^{2} V(z)}{\partial z_{\mu} \partial \bar{z}_{\nu}} d z_{\mu} d \bar{z}_{\nu}
$$

(in the sense of Schwartz) is positive semidefinite. As $V(z)$ does not depend upon $\mathfrak{y}$ we have

$$
\frac{\partial^{2} V(\mathfrak{z})}{\partial z_{\mu} \partial \bar{z}_{\nu}}=\frac{\partial^{2} V(\mathfrak{x})}{\partial x_{\mu} \partial x_{\nu}} .
$$

Also $d z_{\mu} d \bar{z}_{\nu}=d x_{\mu} d x_{\nu}+d y_{\mu} d y_{\nu}+i\left(d y_{\mu} d x_{\nu}-d x_{\mu} d y_{\nu}\right)$. Therefore

(7) Theorems 11.2 and 11.3 were first obtained together with other interesting results on tube domains by Lelong [2]. Convex tube domains also play a role in Bochner-Martin [1], Hitotumatu [1], Bremermann $[1 ; 3 ; 5]$. 


$$
\sum \frac{\partial^{2} V(\mathfrak{z})}{\partial z_{\mu} \partial \bar{z}_{\nu}} d z_{\mu} d \bar{z}_{\nu}=\sum \frac{\partial^{2} V(\mathfrak{x})}{\partial x_{\mu} \partial x_{\nu}}\left(d x_{\mu} d x_{\nu}+d y_{\mu} d y_{\nu}\right) .
$$

The latter form is positive semidefinite if and only if $\sum\left(\partial^{2} V(\mathfrak{x}) / \partial x_{\mu} \partial x_{\nu}\right) d x_{\mu} d x_{\nu}$ is positive semidefinite, and that is the case if and only if $V(\mathfrak{x})$ is convex (according to 9.5 ).

11.3. A tube domain $T_{B} \subset C^{n}$ is pseudo-convex if and only if its basis $B \subset R^{n}$ is convex.

$T_{B}$ is pseudo-convex if and only if $-\log \delta_{T}(z)$ is plsh. in $T_{B}$. Now obviously $\delta_{T}(\mathfrak{z})$ does not depend upon $\mathfrak{y}$ and we have $\delta_{T}(\mathfrak{z})=\delta_{B}(\mathfrak{x})$. $\left(\delta_{B}(\mathfrak{x})\right.$ the distance function of the domain $B$.) As we have just proved, $-\log \delta_{T}(z)$ is pseudo-convex in $T_{B}$ if and only if $-\log \delta_{B}(\mathfrak{x})$ is convex in $B .-\log \delta_{B}(\mathfrak{x})$ is convex if and only if $B$ is convex (9.20). Thus $T_{B}$ is pseudo-convex if and only if $B$ is convex.

11.4. To any convex domain $B \subset R^{n}$ we can associate $T_{B}=\{\mathfrak{z} \mid \mathfrak{x} \in B \wedge \mathfrak{q}$ arbitrary $\} \subset C^{n}$. To any function $U(\mathfrak{x})$, convex in $B$, we can associate the function $V(\mathfrak{z})=U(\mathfrak{x})$, defined in $T_{B}$.

$T_{B}$ is pseudo-convex according to 11.3 and $V(z)$ is plsh. in $T_{B}$ according to 11.2 .

Any theorem that is true for all plsh. functions and all pseudo-convex regions is in particular true for $T_{B}$ and $V(z)$. If we are able to conclude that the statement is also true for the restriction of $V(z)$ to $B$ and $T_{B}$ to $B$, we have derived the corresponding theorem for convex functions and domains.

For instance the upper envelope of a bounded family of plurisubharmonic functions is a plurisubharmonic function. We want to conclude: The upper envelope of a bounded family $\left\{U_{\alpha}(\mathfrak{x})\right\}$ of convex functions $U_{\alpha}(\mathfrak{x})$, defined in $B$, is a convex in $B$. We define $V_{\boldsymbol{\alpha}}(z)=U_{\boldsymbol{\alpha}}(\mathfrak{x})$, then $V_{\boldsymbol{\alpha}}(z)$ is plurisubharmonic in $T_{B}$. Hence the upper envelope $V(z)$ of the family $\left\{V_{\alpha}(z)\right\}$ is plurisubharmonic in $T_{B}$. It does not depend on the imaginary part of $z$. Therefore its restriction is convex in $B$. The restriction of $V(z)$ is the upper envelope of $\left\{U_{\alpha}(\mathfrak{x})\right\}$. Hence the upper envelope of $\left\{U_{\alpha}(z)\right\}$ is convex.

We cannot always conclude in this way. For instance any plurisubharmonic function can be approximated by plurisubharmonic functions that are arbitrarily differentiable. If we want to derive the corresponding theorem for convex functions, we have the difficulty that our theorem for plurisubharmonic functions ensures us only the existence of an arbitrarily differentiable approximating plurisubharmonic function but does not ensure us that this function does not depend upon the imaginary parts. Thus we cannot simply conclude that the restriction is convex.

Thus the possibility of proving corresponding theorems by this "restriction principle" depends upon the logical structure of the theorem, in particular if the theorem contains "existence" quantification.

12. "Extended class" of functions.

12.1. Given a domain in the $z$-plane (with a sufficiently well behaved 
boundary) and continuous boundary values. Then there does exist a function harmonic in the domain and assuming the given boundary values.

In several variables this is no longer true. Given on the boundary of a domain continuous boundary values. There does in general not exist a function pluriharmonic in the domain that assumes the given boundary values.

Even if the boundary values are prescribed only on the $n$-dimensional "distinguished boundary surface" (Bergman $[1 ; 4]$ ), of a $2 n$-dimensional domain (with a distinguished boundary surface), the boundary value problem can, in general, not be solved with pluriharmonic functions. To solve arbitrary continuous boundary values, prescribed on a distinguished boundary surface, Bergman has extended the class of pluriharmonic functions to the "extended class" (Bergman $[4 ; 6]$ ).

12.2. A very handy class of a domain with a distinguished boundary surface-though not containing the most general type-are the "analytic polyhedrons."

An analytic polyhedron is a domain of the form

$$
P=\left\{z|z \in D \wedge| \chi_{1}(z)|<1 \wedge \cdots \wedge| \chi_{k}(z) \mid<1\right\}, \quad k \geqq n,
$$

where $D$ is a region and $\chi_{1}(\xi), \cdots, \chi_{k}(\xi)$ are holomorphic in $D$ and $P \subset \subset D$. Its distinguished boundary surface is the union of all intersections of $n$ different hypersurfaces

$$
\left\{z|z \in \bar{P} \wedge| x_{v_{1}}(z) \mid=1\right\} \cap \cdots \cap\left\{z|z \in \bar{P} \wedge| \chi_{v_{n}}(z) \mid=1\right\} .
$$

The boundary of $P$ consists of $k 2 n-1$ dimensional hypersurfaces $\sigma_{j}$;

$$
\partial P=\bigcup_{j=1}^{k} \sigma_{i} ; \quad \sigma_{i}=\left\{z|z \in \bar{P} \wedge| \chi_{i}(z) \mid=1\right\} .
$$

The boundary of $\sigma_{j_{1}}$ consists of (at most) $k 2 n-2$ dimen'sional surfaces,

$$
\sigma_{i_{1}}=\bigcup_{j_{2}-1}^{k} \sigma_{j_{1} j_{2}}
$$

$\left(\sigma_{j_{1} j_{2}}\right.$ may be empty for certain $\left.j_{2}\right)$,

$$
\sigma_{i_{1} j_{2}}=\left\{z|z \in \bar{P} \wedge| \chi_{i_{1}}(z)|=1 \wedge| \chi_{i_{2}}(z) \mid=1\right\} .
$$

The boundary of $\sigma_{j_{1} j_{2}}$ is $\bigcup_{j_{2}=1}^{k} \sigma_{j_{1}, j_{2}, j_{3}}$ etc. We get finally $\sigma_{j_{1} \cdots j_{n}}$, and the union of all $\sigma_{j_{1} \cdots j_{n}}$ is the "distinguished boundary surface" of $P$.

If the functions $\chi_{1}(z) \cdots \chi_{k}(z)$ are sufficiently well behaved, each $\sigma_{j_{1}} \cdots j_{\text {, }}$ is at most $2 n-\nu$ dimensional and in particular the distinguished boundary surface is $n$-dimensional. The maximum principle holds for $P$ and the distinguished boundary surface and the class of functions that are holomorphic in $\bar{P}$.

12.3. The convex analogue to the analytic polyhedrons are the convex polyhedrons. 
An analytic polyhedron we can write in the form

$$
P=\left\{z|z \in D \wedge \log | \chi_{1}(z)|<0 \wedge \cdots \wedge \log | \chi_{k}(z) \mid<0\right\} .
$$

To the pluriharmonic functions correspond the linear functions as we have seen in $\$ 9$. Therefore we obtain that

$$
P=\left\{\mathfrak{x} \mid \mathfrak{x} \in B \wedge l_{1}(\mathfrak{x})<0 \wedge \cdots \wedge l_{k}(\mathfrak{x})<0\right\}
$$

corresponds to the analytic polyhedron. Here the condition $\mathfrak{r} \in B$ is obsolete, we obtain:

$$
P=\left\{\mathfrak{x} \mid l_{1}(\mathfrak{x})<0 \wedge \cdots \wedge l_{k}(\mathfrak{x})<0\right\}, \quad k \geqq n .
$$

Such a domain is a convex polyhedron.

The boundary consists of at most $k n-1$ dimension plane segments. $\partial P=\bigcup_{j=1}^{k} \sigma_{j}$. The boundary of each segment $\sigma_{j_{1}}$ consists of at most $k n-2$ dimensional plane segments $\sigma_{j_{1} j_{2}}$, etc. The "distinguished boundary surface" consists of a finite number of points, the "corners" of our polyhedron. The maximum principle holds for $P$ and its distinguished boundary surface and the class of linear functions as well as for the class of functions that are convex in $\bar{P}$.

12.4. Bergman's "extended class" (Bergman [4]). Bergman considers the case of 2 variables. Here the distinguished boundary surface is two-dimensional.

Each $\sigma_{j}=\left\{z|z \in \bar{P} \wedge| \chi_{j}(z) \mid=1\right\}$ is the union of the set of analytic surfaces:

$$
F_{j}^{\left(\vartheta_{j}\right)}=\left\{z \mid z \in \bar{P} \wedge \chi_{j}(z)=e^{i \vartheta_{i}}\right\}, \quad 0 \leqq \vartheta_{i} \leqq 2 \pi .
$$

The boundary of each $F_{j}^{\left(v_{i}\right)}$ lies on the distinguished boundary surface. Therefore we have continuous boundary values, prescribed on the boundary of $F_{j}^{\left(\boldsymbol{\theta}^{j}\right)}$.

As $F_{j}^{\left({ }_{j}\right)}$ is a 2-dimensional analytic surface, we can solve the boundary value problem and determine that function that is harmonic on $F_{j}^{\left({ }^{*}\right)}$ and takes the given boundary values. Doing this for every $F_{j}^{\left({ }_{j}\right)}$ we have our function uniquely defined on the whole boundary of $P$.

The next step is to define the function of the extended class in $P$. Bergman has used two different methods (Bergman [4], p. 526). (1) Take that (uniquely determined) harmonic function that takes the prescribed boundary values on $\partial P$, the boundary of $P$. (2) Select one fixed $\chi_{j_{0}}(z)$, and define

$$
\tau_{p, v}=\left\{z \mid z \in P \wedge \chi_{i_{0}}(z)=\rho e^{i \vartheta}\right\} .
$$

Then the union of all $\tau_{\rho, \vartheta}$, for $0 \leqq \rho<1,0 \leqq \vartheta \leqq 2 \pi$, is $P$. Each analytic surface $\tau_{\rho, \vartheta}$ has its boundary on $\partial P$. We can similarly, as above, determine that harmonic function (harmonic on $F_{j_{0}}(\rho, \vartheta)$ ) that assumes the values on the boundary of $F_{j_{0}}(\rho, \vartheta)$. Thus we obtain a function, uniquely defined for every point in $P$. 
By selecting a different $\chi_{j_{0}}(z)$ we would have obtained a different function. Thus there are $k$ different possibilities, $k$ different "extended classes."

12.5. Let us interpret this procedure in the convex case.

In the case of one real variable a convex domain is an interval. The boundary value problem can trivially be solved with a linear function (compare 12.1).

In the case of two variables it is in general quite impossible, of course, to solve the boundary value problem with boundary values prescribed on the whole boundary with linear functions (compare 12.1).

However, given a triangle and boundary values at its "distinguished boundary surface," that is its three corners. By three values a linear function is just uniquely determined.

If we have a polygon with $n$ corners, however, we can, in general, not find a linear function assuming the given $n$ values. We have the situation that corresponds to the case of boundary values prescribed on the distinguished boundary surface of an analytic polyhedron.

Let us now interpret the construction of a function of the "extended class" in the convex case.

Corresponding to the $C^{2}$ is the $R^{2}$ and to an analytic polyhedron corresponds a convex polyhedron $P$. On every side $\sigma_{j}$ of the polyhedron we can determine that function that is linear on the side and that takes the prescribed values in the corners $\sigma_{j_{1} j_{2}}$.

Thus we obtain boundary values on the whole boundary of $P$. To define our function of the "extended class" we have corresponding to 12.4 the cases: (1) Take that harmonic function that assumes the values on $\partial P$. (2) We select one of the sides $\sigma_{j_{0}}$ of the polyhedron and cover with its parallels $\tau_{p}$ the whole domain $P$. The end points of each segment $\tau_{p}$ lie on the boundary of $P$. We can now determine that function, linear on $\tau_{\rho}$, that assumes the boundary values in the end points of $\tau_{\rho}$. Thus we obtain a function defined throughout $P$.

By selecting a different side $\sigma_{j}$ we would have obtained a different function. Thus there are $k$ different possibilities, $k$ different "extended classes."

12.6. In the case of a convex polyhedron we have also another possibility of defining an "extended class" to solve our boundary value problem.

We take the upper envelope of all linear functions that are smaller or equal to the given boundary values. This envelope function is a convex function (compare 9.12). Also, as it can easily be seen, it assumes the prescribed (finitely many) boundary values. This envelope function can be obtained as the upper envelope of at most $k$ different linear functions if we have $k$ corners. This function is also equal to the upper envelope of all convex functions that are smaller or equal to the given boundary values.

12.7. The correspondence between convexity and pseudo-convexity suggests proceeding in the case of analytic polyhedrons in a similar way. 
We can consider the class of all pluriharmonic functions or plurisubharmonic functions, respectively, that are smaller than or equal to the given boundary values on the distinguished boundary surface. The upper envelope is a plurisubharmonic function, because of 9.12 and because the maximum principle holds for analytic polyhedra, its distinguished boundary surface and the class of plurisubharmonic functions (12.2).

We conjecture: This envelope function assumes the given boundary values if the boundary is sufficiently "well behaved."

We shall take up these heuristic ideas in a further paper.

\section{BIBLIOGRAPHY}

\section{N. Aronszajn}

1. Theory of reproducing kernels, Trans. Amer. Math. Soc. vol. 68 (1950) pp. 337-404.

H. BehnKe and E. Pesche

1. Die Konvexität in der Elementargeometrie und in projektiven Räumen, Semesterberichte Münster vol. 5 (1934).

H. BEHNKE and F. SOMMER

1. Über die Voraussetzungen des Kontinuitätssatzes, Math. Ann. vol. 121 (1950) pp. 356-378. H. BEHNKE and K. STEIN

1. Konvergente Folgen von Regularitätsbereichen und die Meromorphiekonvexität, Math. Ann. vol. 116 (1938) pp. 204-216.

2. Die Konvexität in der Funktionentheorie mehrerer Veränderlichen, Mitteilungen der Mathematischen Gesellschaft in Hamburg vol. 8 (1940) pp. 34-81.

3. Der Severische Satz ulber analytische Fortsetzung von Funktionen mehrerer Veränderlichen und der Kontinuitätssatz, Annali di Matematica Pura ed Applicata vol. 36 (1954) pp. 297-313.

H. BehNKe and P. Thullen

1. Theorie der Funktionen mehrerer komplexer Veränderlichen, Ergebnisse der Mathematik vol. 3, no. 3, (1934).

S. BERGMAN

1. Über die ausgezeichneten Randflächen in der Theorie der Funktionen von zwei komplexen Verdnderlichen, Math. Ann. vol. 104 (1931) pp. 611-636.

2. Über die Kernfunktion eines Bereiches und ihr Verhalten am Rande, J. Reine Angew. Math. vol. 169 (1933) pp. 1-42 and vol. 172 (1934) pp. 89-128.

3. Sur les fonctions orthogonales de plusieur variables complexes avec les applications d la theorie des fonctions analytiques, Mémorial des Sciences Math., vol. 106, Paris, 1947.

4. Functions of extended class in the theory of functions of several complex variables, Trans. Amer. Math. Soc. vol. 63 (1948) pp. 523-547.

5. The kernel function and conformal mapping, New York, 1950.

S. Bochner and W. T. MARTIN

1. Several complex variables, Princeton, 1948.

T. BONNESEN and W. FENCHEL

1. Theorie der konvexen Körper, Ergebnisse der Mathematik vol. 3 (1934).

N. BOURBAKI

1. Espaces vectoriels topologiques, Book V, Actualités scientifiques et Industrielles, no. 1189, 1953.

H. J. BREMERMANN

1. Die Charakterisierung von Regularitätsgebieten durch pseudokonvexe Funktionen, Schriftenreihe des Math. Inst. Münster vol. 5 (1951).

2. Holomorphic continuation of the kernel function and the Bergman metric in several com- 
plex variables, Lectures on Functions of a Complex Variable, Ann Arbor, Mich., 1955, pp. 349-383.

3. Die Holomorphie hüllen der Tuben- und Halbtubengebiete, Math. A.nn. vol. 127 (1954) pp. 406-423.

4. Über die Äquivalenz der pseudo-konvexen Gebiete und der Holomorphie-gebiete im Raum von $n$ komplexen Veränderlichen, Math. Ann. vol. 128 (1954) pp. 63-91.

5. On the conjecture of the equivalence of the plurisubharmonic functions and the Hartogs functions, Math. Ann. vol. 131 (1956).

6. Note on plurisubharmonic and Hartogs functions, Proc. Amer. Math. Soc. (to appear).

7. Holomorphic functionals and complex convexity in Banach spaces, to appear.

H. Cartan and P. Thullen

1. Regularitäts- und Konvergenz-bereiche, Math. Ann. vol. 106 (1932), pp. 617-647.

S. Hitotumatu

1. Note on the envelope of regularity of a tube domain, Proc. Japan Acad. vol. 26 (1950) pp. 21-25.

\section{P. LELONG}

1. Les fonctions plurisousharmoniques, Ann. Ecole Norm. vol. 62 (1945) pp. 301-345.

2. La convexite et les fonctions analytiques de plusieur variables complexes, J. Math. Pures Appl. vol. 31 (1952) pp. 191-219.

3. Domaines convexes par rapport aux fonctions plurisousharmoniques, Journal d'Analyse Mathématique vol. 2 (1952-1953) pp. 178-208.

4. Fonctions plurisousharmoniques; mesures de Radon associees. Application aux fonctions analytiques, Colloque sur les fonctions de plusieur variables, Bruxèlles, 1953, pp. 21-40. MARSTON MORSE

1. Topological methods in the theory of functions of a complex variable, Princeton, 1947.

Z. NEHARI

1. On weighted kernels, Journal d'Analyse Mathématique vol. 2 (1952-1953) pp. 126-149 F. NORGUET

1. Sur les domaines d'holomorphie des fonctions uniformes de plusieur variables complexes. K. ORA (Passage du local au global), Bull. Soc. Math. France vol. 82 (1954) pp. 137-159.

1. Sur les fonctions analytiques de plusieur variables, VI, Domaines pseudo-convexes, Tôhoku Math. J. vol. 49 (1942) pp. 15-52.

2. Sur les fonctions analytiques de plusieur variables, IX, Domaines finis sans point critique T. RADO interieur, Jap. J. Math. vol. 23, (1953) pp. 97-155.

1. Subharmonic functions, Ergebnisse der Mathematik, no. 1, vol. 5 (1937).

L. SCHWARTZ

1. Theorie des distributions, Paris, 1950.

P. THULlen

1. Die Regularitätshilllen, Math. Ann. vol. 106 (1932) pp. 64-76.

Institute for Advanced Study,

Princeton, N.J. 